\title{
Study of a Flame Kernel Evolution in a Turbulent Mixing Layer Using LES with a Laminar Chemistry Model
}

\author{
Agnieszka Wawrzak ${ }^{1} \cdot$ Artur Tyliszczak $^{1}$ iD
}

Received: 17 October 2019 / Accepted: 27 April 2020 / Published online: 22 May 2020

(c) The Author(s) 2020

\begin{abstract}
Temporal evolution of an ignition kernel in a spark ignited turbulent hydrogen-air mixing layer is studied using the large eddy simulation approach with an implicit treatment of the reaction source terms. The applied numerical code is based on a high-order compact difference approximation combined with a weighted essentially non-oscillatory scheme, which provide an accurate resolution of the small scale phenomena. The spark is modelled by an energy deposition model coupled with the enthalpy equation. Since the fuel and oxidiser streams move in the opposite directions the flow is dominated by shear stresses and vortical structures. The ignition follows three different scenarios depending on the spark location. An interaction between the developing flame kernel and large turbulent structure is analysed starting from the energy transfer up to a fully reacting state. The size and shape of the flames are correlated with the initial ignition scenario. A 3D visualisation of the transient position of the flame kernel shows its different spatio-temporal behaviour. It is observed that the flame can stay close to the initial position and spread equally in all directions or it can move far from the initial location and follow the evolving flow field. In the latter scenario two separate sub-stages are convincingly identified. Concerning the ignition probability $\left(P_{i g n}\right)$, when the spark is located in the immediate vicinity of the mixing layer, the $P_{i g n}$ on the fuel-rich side is higher. On the other hand, when the ignition occurs far from the mixing layer, the $P_{i g n}$ is significantly larger on the lean side. Unlike the local composition the impact of the strain rate of the velocity field was found to have very limited impact on the $P_{i g n}$.
\end{abstract}

Keywords Spark ignition · Kernel evolution · Mixing layer · Ignition probability

\section{Introduction}

The complete understanding of the turbulent flame ignition is extremely important both from the fundamental as well as practical point of view. Safety and efficiency of technical combustion devices has been getting attention for last decades. A large number of industrial burners, engines and aircraft combustors are based on non-premixed systems

Artur Tyliszczak

atyl@imc.pcz.pl

1 Department of Thermal Machinery, Czestochowa University of Technology, Czestochowa, Poland 
and combustion initiation through an externally introduced energy source (e.g. an electric spark, laser, plasma). Further development of these devices is a challenging task and it requires more experimental and numerical works on the ignition process and flame kernel evolution. A considerable amount of research in this field has been done to date but no univocal conclusions have been found. Most of these studies focus on spark ignition of jets (Birch et al. 1981; Smith et al. 1988; Ahmed and Mastorakos 2006; McCraw et al. 2007; Lacaze et al. 2009; Chen et al. 2017; Zhang et al. 2019), counter-flows (Ahmed et al. 2007) and mixing layers (Chakraborty et al. 2007; Chakraborty and Mastorakos 2008; Ahmed and Mastorakos 2016; EidiAttarZade et al. 2017; Wawrzak and Tyliszczak 2018, 2019). Due to simple configurations, the analysis of these problems offers a deep insight into physics of the flame/turbulence interactions. Strongly unsteady phenomena occurring in the igniting flows including the flame kernel formation, its propagation and stabilisation are of current interest for Computational Fluid Dynamics (CFD) community. The fundamental findings on the ignition phenomenon, discussion of its probabilistic nature, and modelling aspects for gaseous and two-phase flows were presented in review papers by Mastorakos (2009, 2017). In the following, we focus only on some selected problems that are crucial for the present work.

Spark ignition in axisymmetric turbulent jets was studied experimentally by Birch et al. (1981) and Smith et al. (1988) in the 1980s. These early experiments demonstrated that fluctuations of the mixture fraction are critical for the flame kernel generation process when the temperature rises and chemical reactions proceed. The results showed that the probability of successful flame kernel generation $\left(P_{k e r}\right)$ and probability of finding flammable mixture $\left(P_{f}\right)$ at the centreline of the jets were approximately equal, $P_{k e r} \approx P_{f}$. Generation of the flame kernel does not necessarily mean the successful ignition, which is considered as a four-step process, i.e. energy deposition $\rightarrow$ flame kernel formation $\rightarrow$ propagation $\rightarrow$ stabilization. It was observed that the probability of ignition $\left(P_{i g n}\right)$ can be lower than $P_{k e r}$ (Smith et al. 1988). In the jet type flows the kernel formation is regarded as the process, that happens from the time moment of spark initiation up to the time when the downstream edge of the flame starts propagation with the flow and along the radial directions. Then, two different scenarios are possible: (1) a whole flame moves with the flow direction (blow-off); (2) its upstream edge stays for a short time at the initial location and begins the propagation against the incoming reactants - the successful ignition with the flame stabilized as lifted one or attached.

More recently, the differences between $P_{f}, P_{k e r}$ and $P_{i g n}$ were reported by Ahmed et al. (2007) in a counter-flow configuration. They showed that $P_{k e r}<P_{f}$ resulted from the fact that in a region of strong mixing, the flame kernel could have been quenched due to local strain effects or large turbulent fluctuations. Hence, even if the flame was initiated at favourable mixture conditions, the probability of ignition was lower, i.e. $P_{i g n}<P_{k e r}<P_{f}$. One should assume that the same situations can be observed for jet type flames. It is worth to note that Ahmed et al. (2007) observed ignition in locations of unfavourable mixture composition, i.e. in the places with $P_{f}=0$. Their results showed that even when the spark was initiated outside of the ignitable region (e.g. at very lean/rich mixtures) the heat and radicals could be convected towards flammable regions and initiate the flame there. In such cases, the turbulent structures seem to be the most important, as on the one hand their presence may lead to enhanced mixing promoting the ignition process, while on the other, if the turbulence level is too high, the flame kernel can be quenched. In fact, depending on the spark location with respect to a vortical structure, three significantly different ignition scenarios (two successful and one failed) can be identified (Wawrzak and Tyliszczak 2019). It was found that in the case of successful ignition the initial scenario directly affects further 
flame development phase. Worth noting is that not only the size and energy of the sparks can have impact on the success of ignition but also the size and strength of the turbulent structures present in the spark vicinity (Wawrzak and Tyliszczak 2018).

The spark ignition process in a methane jet with particular attention to a spatial distribution of the ignition probability was analysed experimentally by Ahmed and Mastorakos (2006). They considered varying spark parameters and jet velocities and found that: (1) downstream the axial location where the mean mixture fraction drops below the stoichiometric value $\left\langle\xi_{\mathrm{ST}}\right\rangle$ the $P_{i g n}$ decreases significantly; (2) the $P_{i g n}$ is always smaller than one, even along $\left\langle\xi_{\mathrm{ST}}\right\rangle$ isolines; (3) the region of high $P_{i g n}$ decreases with an increasing jet velocity; (4) the $P_{i g n}$ increases for a longer spark duration. The failure of the ignition process was attributed to the lack of the flame kernel or its blow-out at downstream locations, similarly as in Smith et al. (1988). In the work of Ahmed et al. (2007) the failed ignition events associated with the latter situation were observed for the spark locations beyond some critical distance above which the flame could not propagate against the incoming reactants. These findings were partially confirmed by McCraw et al. (2007) in a jet configuration with a pilot flame as the ignition source.

In numerical simulations of the spark ignition process, the spark is modelled one of two ways, i.e. by an energy (or temperature) source term added to the energy equation or by a volume of burning mixture, which, when placed in a flow domain, mimics the flame kernel. The former approach is often coupled both with direct numerical simulations (DNS) (Chakraborty et al. 2007; Chakraborty and Mastorakos 2008) as well as LES (Lacaze et al. 2009; Richardson et al. 2007; Jones and Tyliszczak 2010) with various combustion models. The latter method has been mostly used with LES (Rosiak and Tyliszczak 2016; Triantafyllidis et al. 2009; Tyliszczak et al. 2014; Wawrzak and Tyliszczak 2017). The above mentioned experiment of Ahmed and Mastorakos (2006) was reproduced by Lacaze et al. (2009) using LES combined with the thickened flame combustion model (Colin et al. 2000; Jones and Prasad 2011) with the help of LES and the Eulerian stochastic field method (Valiño 1998), and more recently by Chen et al. (2017) using LES combined with the joint sub-grid PDF approach for partially premixed combustion and Zhang et al. (2019) with the help of LES and the Conditional Moment Closure model. In the first two studies, the spark was defined as the energy source term according to an energy deposition model (Lacaze et al. 2009), while in the latter, it was mimicked by a burning mixture introduced to the flow domains. In all above mentioned papers, the measured size and position (lift-off height) of the flame, as well as its propagation speed were predicted accurately. The study of Chen et al. (2017) revealed that the propagating flame follows a spiral-shaped trajectory. Spatial ignition probability was studied only by Lacaze et al. (2009). It was in good agreement with the measurements in a mixing layer close to the inlet, whereas further downstream from the inlet, the probability was significantly over-predicted.

Recent experimental and numerical research on the spark ignition in a shear-less mixing layer (methane and air) performed in Ahmed and Mastorakos (2016), EidiAttarZade et al. (2017) focused on a V-shaped region of the ignition probability. It was found that the highest $P_{i g n}$ was located around $\xi_{\mathrm{ST}}$. In lean mixtures, it was consistent with the lower flammability limit, whereas in the rich regions, the values of $P_{i g n}$ were reduced to zero before reaching the location of the upper flammability limit.

To summarize, the above findings show that placing the spark in the region where $P_{f}=0$ does not preclude generation of the flame kernel and its subsequent growth. On the other hand, the location of the spark at the point with $P_{f}>0$, where $P_{k e r}>0$, does not guarantee successful ignition. The established flame kernel may grow or may be too weak to propagate against 
the vortices in a highly turbulent flow. Definitely, the ignition process has a stochastic nature which is largely conditioned by flow regimes.

The present paper extends the research performed in Wawrzak and Tyliszczak (2018, 2019). The objective of the study is to analyse the spark ignition in flow conditions dominated by shear stresses and the presence of strong vortical structures. We investigate the mechanism of flame development in a temporally evolving mixing layer formed between streams of fuel $\left(\mathrm{H}_{2} / \mathrm{N}_{2}\right)$ and air flowing in opposite directions, similarly as in Hawkes et al. (2007) for a case of a temporally evolving jet. Usually, the successful spark ignition is considered as a multi-step process in which the main parts are (1) the initial kernel generation, (2) the flame propagation, and (3) stabilisation. In the present configuration, the stable or steady state conditions with a well-defined flame position cannot exist by definition, and therefore, we treat the ignition event as successful when the flame starts to propagate across the domain. We apply the LES method with a detailed chemistry for hydrogen oxidation and 'no model' approach for reaction terms closure, where the chemical source terms are computed directly using the filtered (resolved) temperature and species mass fractions. Such a 'no model' approach is sometimes labelled as 'implicit LES (ILES)' (Duwig et al. 2011), which should not be confused with an implicit approach for sub-filter terms resulting from the filtering operation applied to the convective parts of the governing equations. The two major problems analysed in this paper are: (1) the mechanism of flame kernel formation in the presence of convective forces and strong shear stresses; (2) the impact of turbulent structures on the initial phase of the flame evolution. We focus on two different successful ignition scenarios identified in Wawrzak and Tyliszczak (2019). Furthermore, we put attention to the ignition probability and try to assess its distribution depending on a spark location with respect to an initial shear layer position and a size and strength of the vortical structures formed in its vicinity.

\section{Mathematical Approach}

We consider a low Mach number reacting flow for which the continuity, Navier-Stokes and scalar transport equations, complemented with the equation of state, are defined within the framework of the LES method as:

$$
\begin{gathered}
\partial_{t} \bar{\rho}+\nabla \cdot(\bar{\rho} \widetilde{\mathbf{u}})=0 \\
\bar{\rho} \partial_{t} \widetilde{\mathbf{u}}+(\bar{\rho} \widetilde{\mathbf{u}} \cdot \nabla) \widetilde{\mathbf{u}}+\nabla \bar{p}=\nabla \cdot\left(\boldsymbol{\tau}+\boldsymbol{\tau}^{S G S}\right) \\
\bar{\rho} \partial_{t} \widetilde{Y}_{\alpha}+(\bar{\rho} \widetilde{\mathbf{u}} \cdot \nabla) \widetilde{Y}_{\alpha}=\nabla \cdot\left(\bar{\rho}\left(D_{\alpha}+D_{\alpha}^{S G S}\right) \nabla \widetilde{Y}_{\alpha}\right)+\overline{\dot{w}}_{\alpha} \\
\bar{\rho} \partial_{t} \tilde{h}+(\bar{\rho} \widetilde{\mathbf{u}} \cdot \nabla) \widetilde{h}=\nabla \cdot\left(\bar{\rho}\left(D+D^{S G S}\right) \nabla h\right)+\dot{Q} \\
p_{0}=\bar{\rho} R \widetilde{T}
\end{gathered}
$$

where the bar and tilde symbols denote filtered quantities (Geurts 2004). The variables appearing in the above equations are the velocity vector $\mathbf{u}$, the density $\rho$, the hydrodynamic pressure $p$, the species mass fractions $Y_{\alpha}$ and enthalpy $h$. The term $\dot{Q}$ represents the energy source, whose role is to mimic a real spark. Its definition is discussed later. The 
subscript $\alpha$ represents the index of the species $\alpha=1, \ldots, \mathrm{N}$-species, the symbols $p_{0}$ and $R$ are the thermodynamic pressure and the gas constant, respectively. The quantities $\tau$ and $D_{\alpha}, D$ are the viscous stress tensor and mass and heat diffusivities. The sub-filter tensor is given by $\boldsymbol{\tau}^{S G S}=2 \mu_{t} \mathbf{S}$, where $\mathbf{S}$ is the rate of strain tensor of the resolved velocity field and $\mu_{t}$ is the sub-filter viscosity modelled as in Vreman (2004). The sub-filter diffusivities in Eqs. (3), (4) are computed as $D^{S G S}=\mu_{t} /(\bar{\rho} \sigma)$ where $\sigma$ is the turbulent Schmidt or Prandtl number assumed equal to 0.7 (Branley and Jones 2001; Triantafyllidis and Mastorakos 2009).

\section{1 'No Model’ Approach for the Reaction Terms}

The main difference between LES with the combustion models (e.g. flamelet, thickened flame, conditional moment closure, etc.) and a 'no model' approach relies on treatment of the reaction source terms $\overline{\dot{w}}_{\alpha}$. In the latter approach, the sub-filter scales resulting from filtering of the convective terms are modelled as usually (in this work, the Vreman's

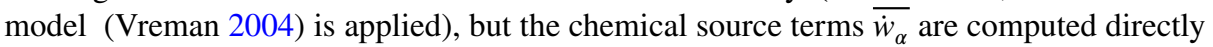
using the filtered variables:

$$
\overline{\dot{w}_{\alpha}(\mathbf{Y}, h)} \approx \dot{w}_{\alpha}(\tilde{\mathbf{Y}}, \tilde{h})
$$

where the impact of the sub-filter unresolved scales is neglected. The 'no model' assumptions would certainly fail in the Reynolds Averaged Navier-Stokes (RANS) framework as the fluctuations in RANS models are large in general. On the other hand, in the laminar flow simulation and in DNS, where all turbulent flow scales are resolved, the 'no model' approach is perfectly valid. Hence, one may assume that for sufficiently dense computational meshes, when the sizes of grid cells are comparable with the Kolmogorov length scale, the use of this method is appropriate, as shown for instance in Duwig et al. (2011), Prasad et al. (2014). The correctness of the 'no model' approach for a flow configuration similar to the one studied in the present work was verified in Wawrzak and Tyliszczak (2018), and here it will be only partially repeated for completeness of the presentation.

\subsection{Numerical Method}

We apply an in-house numerical code (SAILOR) for low Mach number flows with variable density and temperature. The solution algorithm is based on the projection method for pressure-velocity coupling (Tyliszczak 2014, 2016). The time integration is performed by means of an operator splitting approach where the transport in physical space and chemical terms are solved separately. The convective and diffusive parts of the Eqs. (2)-(4) are advanced in time using a predictor-corrector technique with the 2nd order Adams-Bashforth/Adams-Moulton methods. The chemical reactions are computed using CHEMKIN interpreter (http://www.reactiondesign.com/products/open/chemkin.html) and the detailed mechanism of Mueller et al. (1999) involving 9 species and 21 reactions for the hydrogen combustion. The reaction terms are stiff and therefore they are integrated in time applying the VODPK (Brown and Hindmarsh 1989) solver that is well suited for stiff systems. The spatial discretization is performed on half-staggered meshes applying the 6th order compact difference approximation for the Navier-Stokes and continuity equations (Santhanam et al. 2003; Laizet and Lamballais 2009; Tyliszczak 2014). The 5th order WENO scheme (Shu 2003) is used for discretization of the convective terms in the equations for 
the species mass fraction and enthalpy. The applied code has been used and thoroughly verified in previous studies devoted to gaseous flows and flames (Tyliszczak 2015a, b; Rosiak and Tyliszczak 2016; Wawrzak and Tyliszczak 2018).

\subsection{Spark Model}

The spark is modelled by the energy deposition model, where the source term $\dot{Q}$ in Eq. (4) denotes the energy added to the flow through the enthalpy equation. Such a spark does not changes the mixture composition directly. This happens only when chemical source terms activate due to increased temperature level. The source term $\dot{Q}$ has the Gaussian distribution in time and space and is defined as (Chakraborty et al. 2007; Lacaze et al. 2009):

$$
\dot{Q}=\frac{\epsilon}{4 \pi^{2} \sigma_{\mathrm{s}}^{3} \sigma_{t}} \exp \left[-\frac{1}{2}\left(\frac{r-r_{0}}{\sigma_{\mathrm{s}}}\right)^{2}\right] \exp \left[-\frac{1}{2}\left(\frac{t-t_{0}}{\sigma_{t}}\right)^{2}\right]
$$

where $r$ is the radial distance to the centre of the spark $r_{0}, t$ denotes the time and $t_{0}$ is the time when the spark energy reaches the maximum. Three control parameters are the energy deposited $\epsilon, \sigma_{\mathrm{s}}=\Delta_{\mathrm{s}} / a$ and $\sigma_{t}=\Delta_{t} / a$, where $\Delta_{\mathrm{s}}$ and $\Delta_{t}$ are the characteristic size and duration of the spark, respectively. The coefficient $a=4 \sqrt{\ln (10)}$ ensures that $98 \%$ of the deposited energy is present in the domain $\Delta_{\mathrm{s}}^{3} \times \Delta_{t}$ (Lacaze et al. 2009). The spark defined by Eq. (7) has a spherical shape, which does not change in time. The flow surrounding the spark and being inside it does not affect its size. However, when the spark is switched on, its intensity (power) changes in space and time according to Eq. (7) and reaches the maximum at $t=t_{0}$. As will be shown later, before this occurs, an initially spherical flame kernel arising around the spark is significantly torn by the neighbouring vortical structures.

\section{Configuration and Simulation Details}

A sketch of the computational geometry is shown in Fig. 1. It is a rectangular box with the dimensions $L_{x}=0.025 \mathrm{~m}, L_{z}=0.01 \mathrm{~m}$ and $L_{y}=0.04 \mathrm{~m}$ in the streamwise $(x)$, spanwise $(z)$ and transversal $(y)$ directions, respectively. The flow is periodic in the $x$ and $z$-directions while for $y= \pm L_{y} / 2$ the moving walls are assumed. The fuel flows in the upper area and air flows in the lower one, whereas both streams have the same initial temperature of 300 $\mathrm{K}$. The fuel is a mixture of hydrogen and nitrogen with the mass fractions $Y_{\mathrm{H}_{2}}=0.1$ and $Y_{\mathrm{N}_{2}}=0.9$ for which the stoichiometric value of the mixture fraction is $\xi_{\mathrm{ST}}=0.225$. In this work, following the Bilger's definition, the mixture fraction is computed as:

$$
\xi=\frac{\left(Y_{\mathrm{H}}-Y_{\mathrm{H}, \mathrm{o}}\right) / 2 W_{\mathrm{H}}-\left(Y_{\mathrm{O}}-Y_{\mathrm{O}, \mathrm{o}}\right) / W_{\mathrm{O}}}{\left(Y_{\mathrm{H}, \mathrm{f}}-Y_{\mathrm{H}, \mathrm{o}}\right) / 2 W_{\mathrm{H}}-\left(Y_{\mathrm{O}, \mathrm{f}}-Y_{\mathrm{O}, \mathrm{o}}\right) / W_{\mathrm{O}}}
$$

where $Y$ 's are the elemental mass fractions, $W$ 's are the atomic weights and the subscripts, $f$ and $o$, correspond to the fuel and air, respectively.

The initial velocity field is defined by a hyperbolic tangent function as $u(y)=U_{\infty} \tanh (2 y / \delta)$ where $U_{\infty}$ is the free stream velocity and $\delta=2 U_{\infty} /|d u / d y|_{\max }$ is the initial vorticity thickness assumed equal to $\delta=0.5 \mathrm{~mm}$. The Mach number based on the free stream velocity is approximately equal to 0.05 , which justifies the use of low Mach number approximation. The shape factor of the velocity profile $H=\delta^{*} / \delta_{\theta}=2.25$, where 
Fig. 1 Schematic view of the computational configuration (not in scale)

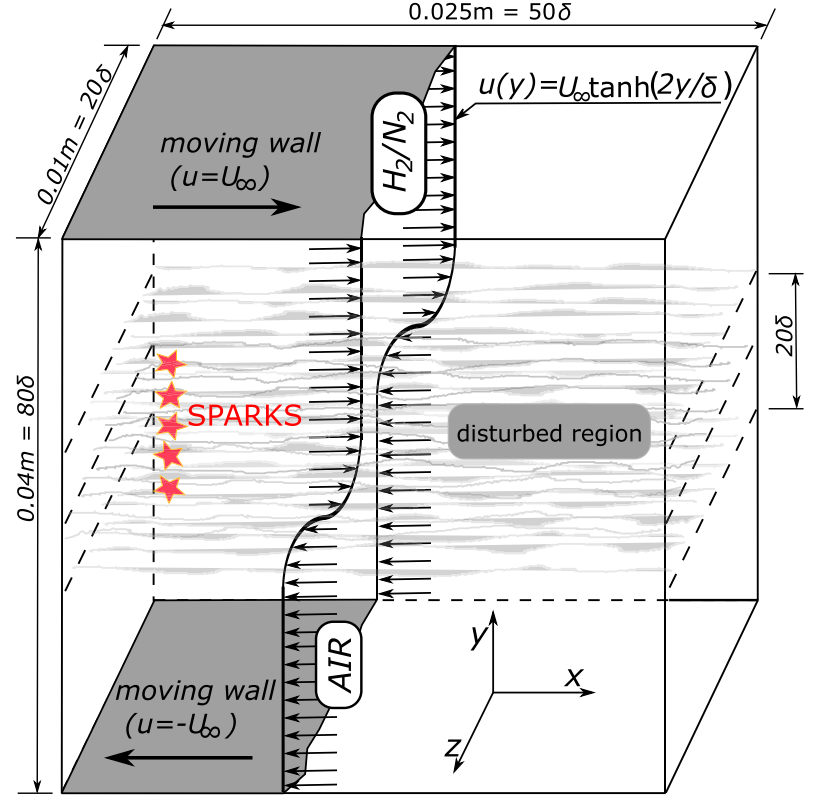

$\delta^{*}=\int_{0}^{\infty}\left(1-U / U_{\infty}\right) \mathrm{d} y$ is the displacement thickness and $\delta_{\theta}=\int_{0}^{\infty} U / U_{\infty}\left(1-U / U_{\infty}\right) \mathrm{d} y$ is the momentum thickness, indicates that the initial velocity profile is typical for a boundary layer in transitional regimes. The Reynolds number is $\operatorname{Re}_{\delta}=U_{\infty} \delta / v_{\text {air }}=600$, which corresponds to jet flows at moderate speeds. The assumed tangent hyperbolic profile reflects very well the velocity profile, which in a jet forms 1-2 diameters downstream a nozzle exit. In the temporally evolving mixing layer the tangent hyperbolic profile was used by Moser and Rogers (1993), among others. In the region of the mixing layer $\pm 10 \delta$ we introduce an isotropic initial disturbance defined through the Passot-Pouquet kinetic energy spectrum, which in terms of a wave number, $k$, is given as (Passot and Pouquet 1987):

$$
E(k)=16 \sqrt{\frac{2}{\pi}} \frac{u^{\prime 2}}{k_{0}}\left(\frac{k}{k_{0}}\right)^{4} \exp \left[-2\left(\frac{k}{k_{0}}\right)^{2}\right]
$$

where $k_{0}$ is an adjustable wave number allowing generation of a turbulent flow field with the required integral length scale $\ell \approx k_{0}^{-1}$ and Taylor length scale $\lambda=\sqrt{\left\langle u^{\prime 2}\right\rangle /\left\langle\left(\partial u^{\prime} / \partial x\right)^{2}\right\rangle}$. Here, the symbol $u^{\prime}$ is the initial velocity fluctuation. The Passot-Pouquet turbulent kinetic energy (TKE) spectrum is known to skip the inertial range and is widely used to represent the flow scales having dissipative character (Martinez et al. 1997). The energy contained in a range $k \in\left[k_{0}, \infty\right]$, i.e. $E_{k_{0} \rightarrow \infty}=\int_{k_{0}}^{\infty} E(k) d k$, is approximately $20 \%$ larger than in the range $k \in\left[0, k_{0}\right]$ and therefore the initial conditions largely depend on the small eddies of the Kolmogorov length scale $\eta$. The Passot-Pouquet spectrum was used to generate the initial conditions proposed by Doom and Mahesh (2009) for auto-ignition studies in a shear-less mixing layer of hydrogen-air mixtures and by Kolla et al. (2016) in research focused on behaviour of a temporally evolving jet in varying Damköhler number regimes. The generator of the initial conditions requires a random set of numbers (seeds), which along with the assumed values of $k_{0}$ and $u^{\prime}$, lead to the velocity fields that follow the same TKE but differ in the spatial distribution of the velocity fluctuations. In this study we consider 16 initial 
velocity fields, S0-S15, whose common global parameters are listed in the Table 1 . For an assumed turbulence intensity $u^{\prime} / U_{\infty}$ equal to $1 \%$, the Reynolds number based on the Taylor length scale of the initial fluctuations is equal to $\operatorname{Re}_{\lambda}=u^{\prime} \lambda / v_{\text {air }}=1.2$ and the Kolmogorov length scale is $\eta \approx \delta / 10$. When the computations start and time passes, the flow develops and larger vortices arise. At the ignition moments, the values of $\operatorname{Re}_{\lambda}$ vary from approximately 30-60, which is of the same order as those used in Hawkes et al. (2007), Doom and Mahesh (2009), Chakraborty and Cant (2011).

\subsection{Verification of the Computational Settings}

The computational mesh is uniform in the periodic directions and compacted towards the centre of the domain in the $y$-direction. The compactness of the grid nodes is defined by a hyperbolic tangent function in such a way that in the vicinity of the mixing layer $(-2 \delta<y<2 \delta)$ the nodes are distributed almost uniformly. Figure 2 shows the computational domain with sample results illustrating the size of the vortical structure (2D contours and 3D iso-surfaces of the vorticity magnitude) and the flame kernel (2D contours of temperature) with respect to the initial shear layer thickness. These results were obtained for the successful ignition event and correspond to the time moment when the spark has been just switched-off and the flame has begun to freely propagate in space. The mesh used in all the simulations consisted of $512 \times 128 \times 256$ nodes in the $x, z$ and $y$-direction, respectively. Compared to the Kolmogorov length scale the cell sizes were equal to: $\eta / \Delta x=0.98, \eta / \Delta z=0.61, \eta / \Delta y_{\min }=1.72$ (at centreline). The mesh resolution slightly changes in the region of the spark placement $(-4 \delta \leq y \leq 4 \delta)$ and at the locations at $y= \pm 4 \delta$ we have $\eta / \Delta y=0.96$, which from the point of view of the LES ensures very good representation of the smallest resolved scales. Note that in the Vreman's sub-grid model (Vreman 2004) the sub-grid viscosity vanishes when a mesh meets DNS requirements. The mesh lines shown in Fig. 2 are plotted every four mesh points in all directions. However, it can be seen that the smallest sub-filter curvatures of the vorticity or temperature isolines are smooth and comparable with the cell sizes.

A grid independence study for a flow configuration similar to the one analysed in the present work were performed in Wawrzak and Tyliszczak (2018). There it was shown that the grid with $512 \times 128 \times 256$ nodes is adequate to well predict the phenomena crucial to ignition, i.e. vortices formation and their evolution at the later time. A convincing argument supporting this statement is provided in Fig. 3 showing the ratio of the sub-filter velocity to the free stream velocity and the ratio of the turbulent viscosity to the molecular one. The presented results have been extracted along the $x$-direction in the central cross-section plane and are conditioned by the stoichiometric mixture fraction, i.e. only these values of $u^{\mathrm{SGS}}$ and $\mu_{T}$ for which $\xi=\xi_{\mathrm{ST}}$ are presented. The sub-filter scale velocity fluctuation was computed based on the sub-grid viscosity as $u^{\mathrm{SGS}}=\frac{\mu_{t}}{\rho C \Delta}$, where $\Delta=(\Delta x \times \Delta y \times \Delta z)^{1 / 3}$ is the local LES filter width and the model constant is $C=0.1$ (Pope 2000). The results presented in Fig. 3 correspond to two specific time instants, $t=1.00 \mathrm{~ms}$ and $t=1.25 \mathrm{~ms}$, which are the times of formation of large scale vortices and their roll-up process, respectively. At these specific time moments the spark energies will reach maximum values. At $t=1.00 \mathrm{~ms}$ and $t=1.25 \mathrm{~ms}$ the Reynolds numbers computed based on the Taylor length scale in the homogeneous $z$-direction

Table 1 List of parameters of the initial velocity fields S0-S15

\begin{tabular}{llllll}
\hline Case & $\delta(\mathrm{mm})$ & $R e_{\delta}$ & $u^{\prime} / U_{\mathrm{inf}}$ & $R e_{\lambda}$ & $\eta(\mathrm{mm})$ \\
\hline S0-S15 & 0.5 & 600 & 0.01 & 1.2 & $\delta / 10$ \\
\hline
\end{tabular}



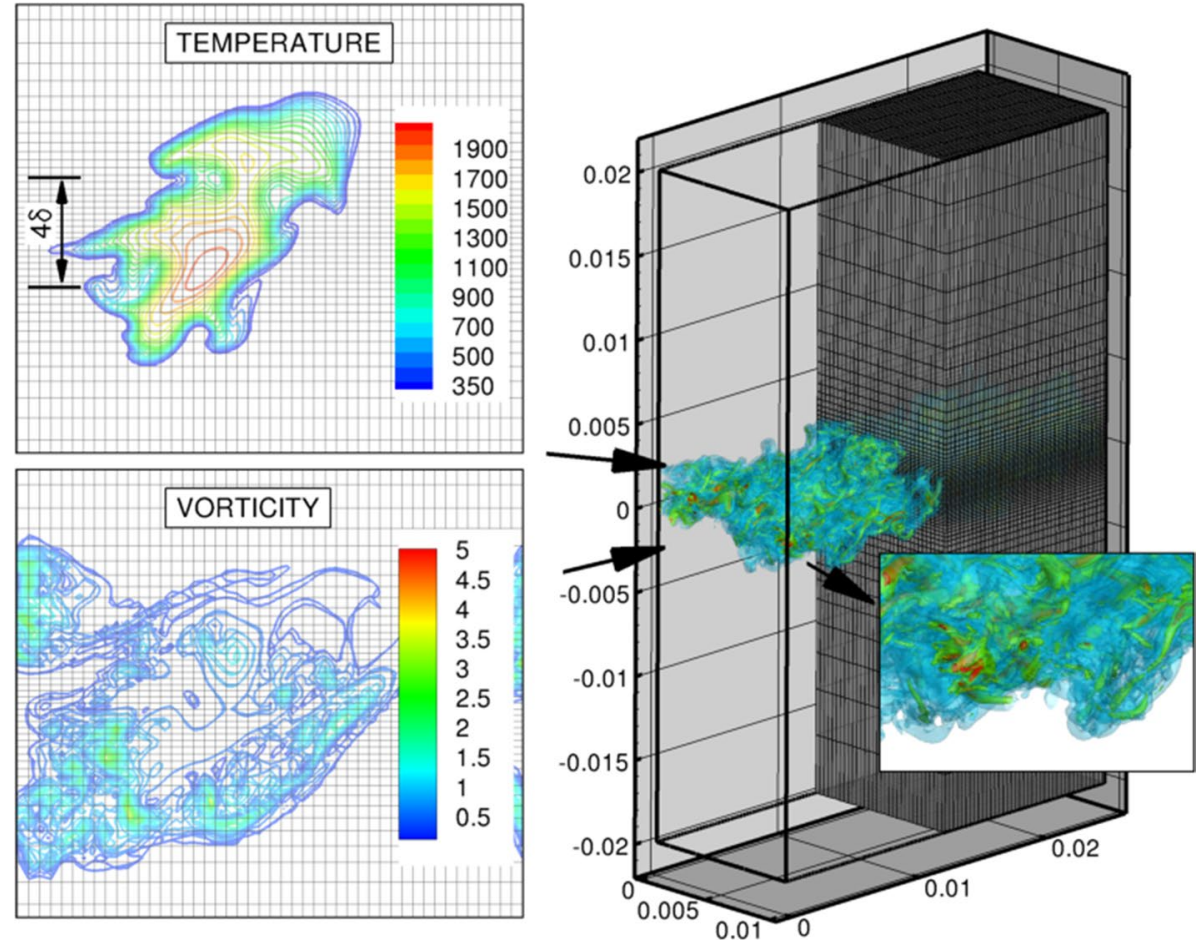

Fig. 2 Computational domain with the mesh and the vorticity magnitude iso-surfaces and the temperature and vorticity magnitude contours in the central ' $x-y$ ' cross-section plane. The mesh points are drawn every four

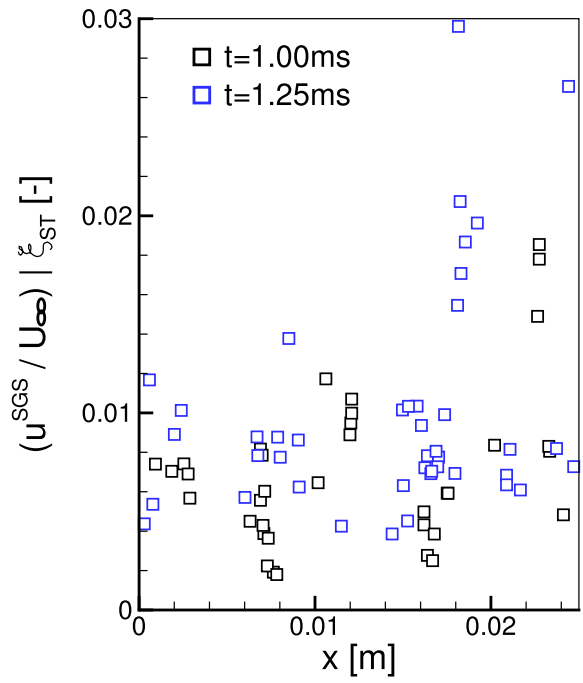

(a)

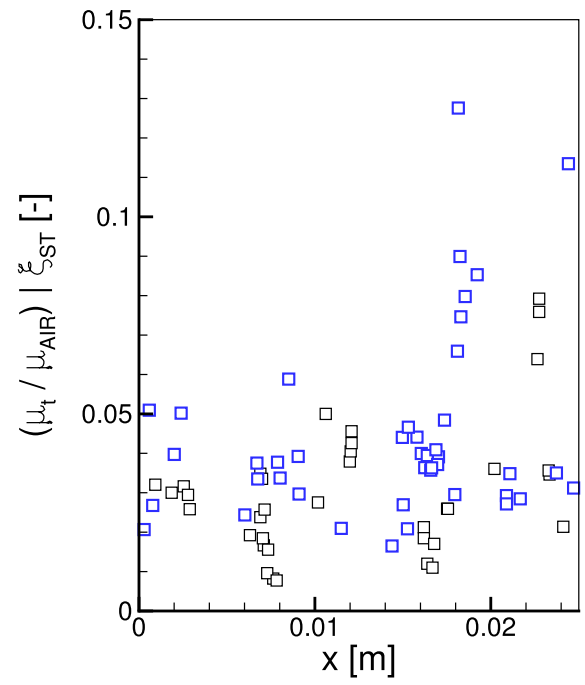

(b)

Fig. 3 Ratio of the sub-filter fluctuations to free stream velocity (a) and ratio of the turbulent to molecular viscosity (b) conditioned by the stoichiometric mixture fraction 
in the region $y / \delta= \pm 10$ are equal to $\operatorname{Re}_{\lambda_{z}}=29$ and $\operatorname{Re}_{\lambda_{z}}=58$. This means that the flow significantly evolved and the vortices grew noticeably compared to their initial sizes. Nevertheless, all flow structures are accurately represented by the computational mesh. This can be deduced from the fact that the sub-filter velocity fluctuations are very small and the turbulent viscosity is almost two orders of magnitude smaller than the molecular viscosity, as can be seen in Fig. 3. In the whole domain and during the entire simulation time, the ratio of the subfilter kinetic energy estimated as $k^{\mathrm{SGS}}=\frac{\mu_{T}^{2}}{(\rho C \Delta)^{2}}$ to the total turbulent kinetic energy is less than 0.1 in all computations performed in this paper. Hence, according to Pope's criterion (Pope 2004) the used mesh provides proper resolution from the point of view of LES needs. It holds not only taking into account the initially assumed flow field but also during its later evolution. Nevertheless, to show the level of dependence of the results on the mesh density, the solutions obtained using two additional meshes consisting of different number of grid points in the ' $x-y$ ' plane, i.e. $256 \times 192$ and $512 \times 384$ were performed. Concerning the spanwise $(z)$ direction the use of the mesh with 192 nodes virtually does not change the solution. The results obtained from comparative simulations will be presented in Sects. 3.2 and 4.

As discussed above, the computational mesh applied is sufficiently dense for accurate and well resolved LES studies. However, its correctness for the 'no model' approach requires a bit more attention. Figure 4 shows a regime diagram for LES of turbulent combustion modelling proposed by Pitsch and Duchamp De Lageneste (2002). In this plot, particular regions are defined with respect to a local sub-grid Karlovitz number $\left(\mathrm{Ka}^{\mathrm{SGS}}\right)$ and the ratio of the LES filter width and the laminar flame thickness $\left(\delta_{l}\right)$. The subgrid Karlovitz number combines the sub-grid quantities with the chemical scales, and hence, it may serve to verify whether the 'no model' approach method is valid at given mesh resolution. Following Pitsch and Duchamp De Lageneste (2002), the Karlovitz number can be estimated based on the laminar flame thickness, burning velocity $\left(S_{l}\right)$ and sub-grid fluctuations as:

$$
\mathrm{Ka}^{\mathrm{SGS}}=\left(\frac{u^{\mathrm{SGS}}}{S_{l}}\right)^{3 / 2}\left(\frac{\delta_{l}}{\Delta}\right)^{1 / 2}
$$

The flame thickness is roughly estimated as $\delta_{l}=D / S_{l}=2.01 \times 10^{-5} \mathrm{~m}$, where $D=2.512 \times 10^{-5} \mathrm{~m}^{2} / \mathrm{s}$ is the heat diffusion coefficient for the stoichiometric mixture and the burning velocity of diluted hydrogen equals to $S_{l}=1.3 \mathrm{~m} / \mathrm{s}$ ( $\mathrm{Li}$ et al. 2018). Taking into account that $\Delta x=4.88 \times 10^{-5} \mathrm{~m}, \Delta z=7.81 \times 10^{-5} \mathrm{~m}, \Delta y_{\min }=2.79 \times 10^{-5} \mathrm{~m}$ and the

Fig. 4 Combustion regime diagram for LES according to Pitsch and Duchamp De Lageneste (2002)

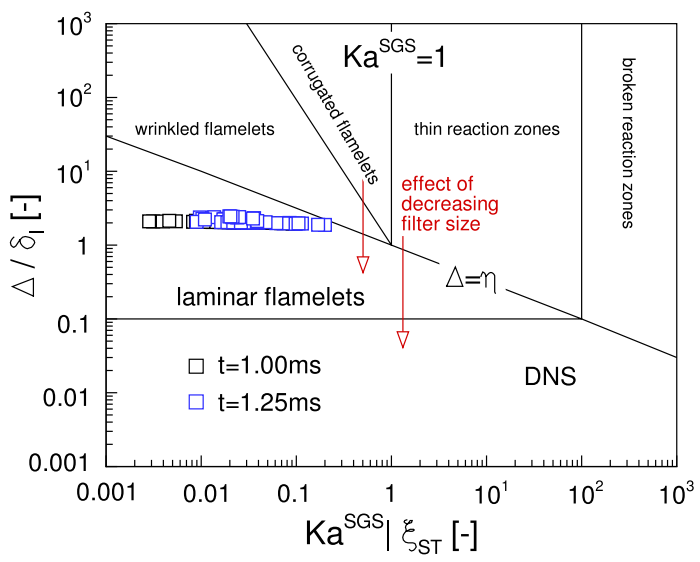


minimum filter width is $\Delta_{\text {min }}=4.73 \times 10^{-5} \mathrm{~m}$, it is clear that the flame front cannot be correctly resolved on the present mesh, even assuming that the above definition of $\delta_{l}$ is too rigorous and the real flame thickness can be even a few times larger (Poinsot and Veynante 2001).

The results shown in Fig. 4 correspond to the ones presented previously in Fig. 3. It can be seen that all points lie in the laminar flamelet regimes, where the turbulent velocity fluctuations are small and accurately captured by the computational mesh $(\Delta \approx \eta)$, and where the flame thickness is smaller than the LES filter width $\left(\Delta>\delta_{l}\right)$. According to Duwig et al. (2011) the 'no model' approach works correctly when the flow inside the LES cells can be treated as in perfectly stirred reactors, which means that it has to be homogeneous and the fluctuations must be small to have no significant impact on chemical reactions. A simple method allowing the assessment of the mixedness level of the reactants seems to be offered by using the mixture fraction gradients $(|\nabla \xi|)$, whose values close to zero indicate the regions of homogeneous mixture or the regions of a pure fuel or oxidiser. Figure 5 presents the contours of $|\nabla \xi| /|\nabla \xi|_{\text {MAX }}$ in the central cross-section plane at the time moments $t=1.00 \mathrm{~ms}$ and $t=1.25 \mathrm{~ms}$. It can be seen that large values occur only in the central part of the domain, where the fuel and oxidiser streams mix and the vortical structures develop. The enlarged part of the figures show the places in which $|\nabla \xi| /|\nabla \xi|_{\text {MAX }}$ changes from zero to one across a thin layer. One can see that this layer is covered by at least 5-6 grid cells, which is certainly sufficient to correctly represent its spatial evolution, especially with the use of high-order discretization method. However, the assumption that within these particular cells the mixture is homogeneous, as would be advisable for the 'no model' approach, is probably slightly exaggerated. Nevertheless, possible modelling errors have the impact only on a very small region of the flow, not necessarily corresponding to the spark location. Thus, neglecting the sub-filter scales in the reaction terms for the simulation of combustion in the present flow configuration should not falsify the underlying physics.

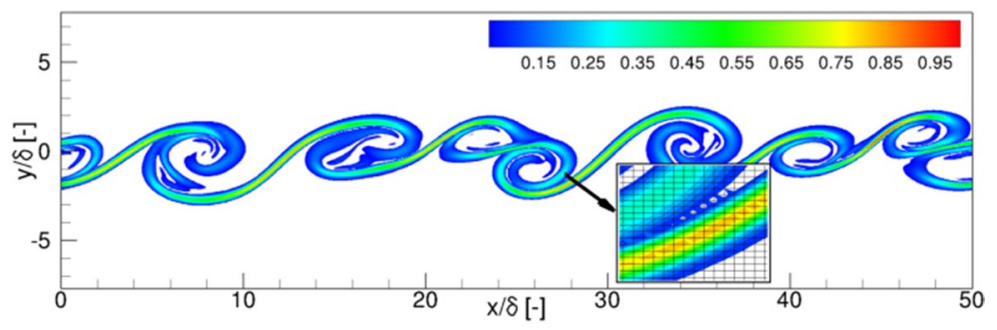

(a) $t=1.00 \mathrm{~ms}$

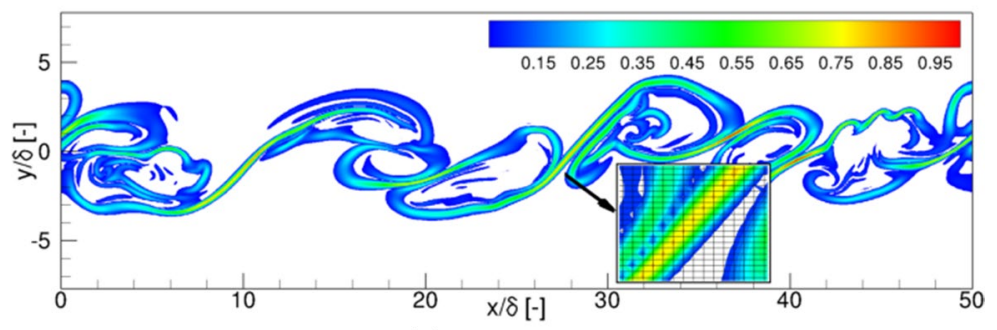

(b) $t=1.25 \mathrm{~ms}$

Fig. 5 Contours of instantaneous values of $|\nabla \xi| /|\nabla \xi|_{\mathrm{MAX}}$ at $t=1.00 \mathrm{~ms}(\mathbf{a}), t=1.25 \mathrm{~ms}(\mathbf{b})$ 


\subsection{Spark Parameters}

We assume the spark with an overall duration time $\Delta_{t}=0.5 \mathrm{~ms}$, total energy $\epsilon=1 \mathrm{~mJ}$ and diameter $\Delta_{\mathrm{s}}=1.5 \mathrm{~mm}=3 \delta$. These parameters are comparable with those used in Ahmed and Mastorakos (2006) for the ignition of a fuel jet and in our previous study (Wawrzak and Tyliszczak 2018) focussed on the importance of the spark parameters. The sparks are placed at $x / \delta=10, z / \delta=\mathrm{L}_{z} / 2=10$ and at various vertical locations: $y / \delta=0, \pm 1, \pm 2, \pm 4$. Note that the spark size is three times larger than $\delta$ that could suggest that its relatively small movement will not result in a significantly different ignition scenario. However, as will be presented later, this supposition turned out to be false. Figure 6 shows the spark locations (the red stars) along with the vorticity magnitude contours and isoline of the stoichiometric mixture (black line) at the selected time instants.

Characteristic phenomena for the temporally evolving mixing layer include continuous growth of the initial disturbances, formation of the vortical structures and their roll-up. Shortly after the simulation starts, at $t=0.5 \mathrm{~ms}$, the fuel and oxidiser streams are still clearly separated and a boundary between them is only slightly waved. The first vortices, which are the results of growth of the most amplified disturbance mode, start to be seen at $t=0.75 \mathrm{~ms}$ (Fig. 6b). Evolution of particular disturbance modes can be analysed using a globally defined integral parameter (Lesieur et al. 1988; Moser and Rogers 1993):

$$
A_{\alpha}=\left[\int_{-\mathrm{L}_{y} / 2}^{\mathrm{L}_{y} / 2} 2 \hat{u}_{x}(\alpha) \hat{u}_{x}^{*}(\alpha) \mathrm{d} y\right]^{1 / 2}
$$

where the variable $\hat{u}_{x}(\alpha)$ is the amplitude of the Fourier mode of the $x$-velocity component and $\hat{u}_{x}^{*}(\alpha)$ is its conjugate value. Figure 7 shows the time evolution of $A_{\alpha}$ of the most amplified mode $\left(A_{1}\right)$ and its subharmonics $\left(A_{1 / 2}\right)$. The maximum of $A_{1}$ occurs at

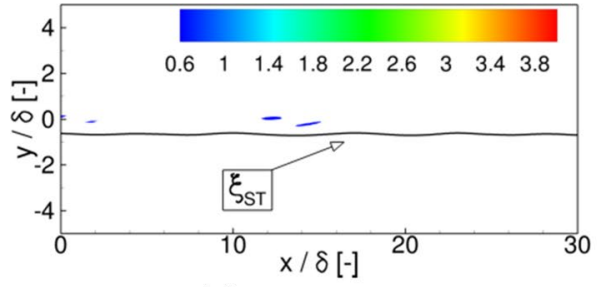

(a) $t=0.50 \mathrm{~ms}$

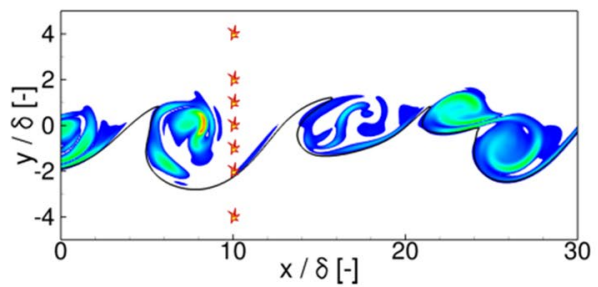

(c) $t=1.00 \mathrm{~ms} \approx t_{A_{1} \mathrm{MAX}}$

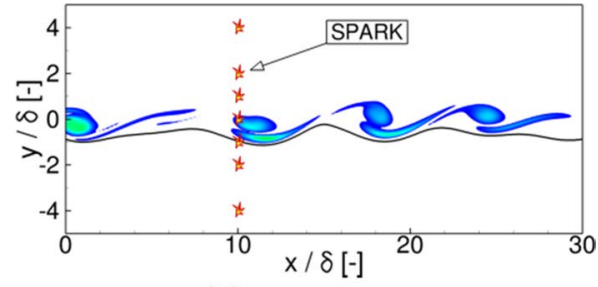

(b) $t=0.75 \mathrm{~ms}$

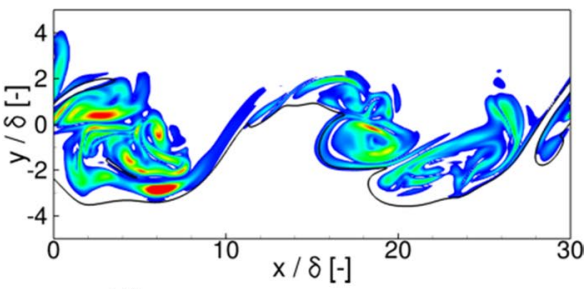

(d) $t=1.25 \mathrm{~ms} \approx t_{A_{1 / 2} \mathrm{MAX}}$

Fig. 6 Contours of instantaneous vorticity magnitude and $\xi_{\mathrm{ST}}$ isoline at various time instants prior to the spark initiation. The results are shown for the case SO 
$t_{A_{1} \mathrm{MAX}} \approx 1.00 \mathrm{~ms}$ and it corresponds to the time instant when the small vortices, visible along the shear layer at $t=0.75 \mathrm{~ms}$, have fully developed and rolled-up. As can be seen in Fig. 6 , at the later time they grow and roll-up when $A_{1 / 2}$ attains the maximum value at $t_{A_{1 / 2} \mathrm{MAX}} \approx 1.25 \mathrm{~ms}$. This scenario agrees very well with the findings of Moser and Rogers (1993). The results presented in Fig. 7 were obtained on three different meshes labelled as M1, M2 and M3. The differences between the solutions obtained on two densest meshes, M2 and M3, are negligible during almost the entire simulation time and are noticeable only from $t=1.75 \mathrm{~ms}$. As will be discussed later, at this time instance, the spark will be already switched-off and the result of the ignition event (successful or failed) and a state of the flame (a size and direction of propagation) will be fully known. Hence, the mesh M2, which is used for all performed simulations is sufficiently dense to correctly predict the mechanism of flame kernel evolution. Concerning the mesh M1, which is four times coarser than M3, it can be seen that its use also ensures a qualitatively good solution. The observed differences are only quantitative. The time at which $A_{1}$ and $A_{1 / 2}$ achieve maxima is predicted very well on all meshes and only their values on the mesh M1 are slightly under-predicted. This, however, can be expected as the content of the resolved energy on the coarser mesh is smaller by definition.

Intuition suggests that for two different spark initiation times, the sparks will be surrounded by vortical structures of different sizes and this will lead to different behaviour of the flame kernel, i.e. to the success or failure of the ignition. Hence, we divided the test cases into two groups depending on spark initiation time. First we considered the situation when the spark was turned on at $t_{\mathrm{sp}}=1.0 \mathrm{~ms} \approx t_{A_{1} \mathrm{MAX}}$ and reached the maximum when the small vortices rolled-up and larger vortical structures were present in the flow at $t_{0}=t_{A_{1 / 2} \mathrm{MAX}}$ (see Fig. $6 \mathrm{c}, \mathrm{d}$ ). In the second case, the spark was turned on at the earlier time, $t_{\mathrm{sp}}=0.75 \mathrm{~ms}$, when the mixing layer was only wrinkled (see Fig. 6b). Most of the attention was devoted to the first situation, while the second one was considered only for the ignition probability analysis. To study this issue we generated sixteen initial flow fields (S0-S15) having the same global characteristics $\left(\operatorname{Re}_{\delta}, \operatorname{Re}_{\lambda}, T_{i}, \eta\right)$ but resulting from a different random seed used to compute the velocity fluctuations, as discussed in Sect. 3.

\subsection{Flow Characteristics Prior to Spark Ignition}

The ignition process depends on the mixture composition, its gradients and the velocity field. In the following sections we focus on two cases corresponding to the initial conditions labelled as S1 and S8, which were selected from all simulations as the representative for highlighting differences in the ignition process. The flow conditions, i.e. the mixture fraction $(\xi)$, the scalar dissipation rate (SDR, $\chi=2 D \nabla \xi \cdot \nabla \xi)$ and module of the strain rate tensor $\left(|S|=\sqrt{2 S_{i j} S_{i j}}\right)$ representing the mixture fraction and velocity gradients, are presented in Table 2. It shows the values for the cases S1 and S8 and also the mean values calculated based on all 16 cases at all spark locations $(x / \delta=z / \delta=10,-4 \leq y / \delta \leq 4)$ at the time $t_{\mathrm{sp}}=1.0 \mathrm{~ms}$. The underlined values correspond to the cases which are analysed in detail in the rest of the paper. In Table 2 the mixture fraction is normalized by its stoichiometric value and the strain rate tensor by its maximum value at the beginning of the simulation $\left(|S|_{\text {MAX }}^{\mathrm{INI}}\right)$. As can be seen the instantaneous vorticity thickness $(\delta(t))$ is approximately three times larger then the initial one. In the case $S 1$ the values of $|S|$ and $\delta(t)$ are slightly higher than for the case S8, however, they are not far from the mean values. Significant changes are found when the spark location changes, especially in the region $-2<y / \delta<2$. There, the strain rate and the SDR for the case S1 are higher than in the case S8. Note that 
Table 2 List of conditions prior to the ignition at the spark centre $(x / \delta=z / \delta=10, y / \delta)$ for the initial velocity field $\mathrm{S} 1, \mathrm{~S} 8$ and the mean values from the cases S0-S15

Fig. 7 Growth of the amplitude disturbances according to Eq. (11). The results are shown for the case S0

\begin{tabular}{|c|c|c|c|c|c|c|}
\hline Case & $t_{\mathrm{sp}}(\mathrm{ms})$ & $\delta(t) / \delta$ & $y / \delta$ & $\xi / \xi_{\mathrm{ST}}$ & $|S| /|S|_{\text {MAX }}^{\text {INI }}$ & $\operatorname{SDR}\left(\mathrm{s}^{-1}\right)$ \\
\hline \multirow[t]{7}{*}{$\mathrm{S} 1$} & \multirow[t]{7}{*}{1.00} & \multirow[t]{7}{*}{3.36} & +4.0 & 4.44 & 0.05 & $1.01 \times 10^{-3}$ \\
\hline & & & +2.0 & 4.16 & 0.21 & $2.34 \times 10^{+1}$ \\
\hline & & & +1.0 & 2.55 & 0.38 & $3.09 \times 10^{+1}$ \\
\hline & & & 0.0 & 1.81 & 0.57 & 1.21 \\
\hline & & & -1.0 & $\underline{2.30}$ & $\underline{0.55}$ & $\underline{1.88 \times 10^{+2}}$ \\
\hline & & & -2.0 & 0.09 & 0.14 & $1.72 \times 10^{-1}$ \\
\hline & & & -4.0 & 0.002 & 0.03 & $7.35 \times 10^{-5}$ \\
\hline \multirow[t]{7}{*}{ S8 } & \multirow[t]{7}{*}{1.00} & \multirow[t]{7}{*}{2.83} & +4.0 & 4.44 & 0.04 & $1.00 \times 10^{-3}$ \\
\hline & & & +2.0 & 4.28 & 0.19 & $6.53 \times 10^{-1}$ \\
\hline & & & +1.0 & 3.88 & 0.38 & 2.83 \\
\hline & & & 0.0 & 0.74 & 0.43 & 1.32 \\
\hline & & & -1.0 & $\underline{1.67}$ & $\underline{0.27}$ & $\underline{4.47 \times 10^{+1}}$ \\
\hline & & & -2.0 & 0.21 & 0.18 & 4.33 \\
\hline & & & -4.0 & 0.002 & 0.02 & $1.03 \times 10^{-4}$ \\
\hline \multirow[t]{7}{*}{$\langle\mathrm{S} 0-\mathrm{S} 15\rangle$} & \multirow[t]{7}{*}{1.00} & \multirow[t]{7}{*}{3.03} & +4.0 & 4.44 & 0.06 & $1.00 \times 10^{-3}$ \\
\hline & & & +2.0 & 3.76 & 0.22 & 3.37 \\
\hline & & & +1.0 & 3.31 & 0.41 & $9.66 \times 10^{+1}$ \\
\hline & & & 0.0 & 2.50 & 0.49 & $7.27 \times 10^{+1}$ \\
\hline & & & -1.0 & 2.04 & 0.41 & $4.28 \times 10^{+1}$ \\
\hline & & & -2.0 & 0.85 & 0.22 & 5.68 \\
\hline & & & -4.0 & 0.003 & 0.04 & $1.54 \times 10^{-4}$ \\
\hline
\end{tabular}

The underline indicates the cases, which are in detail analysed in the paper

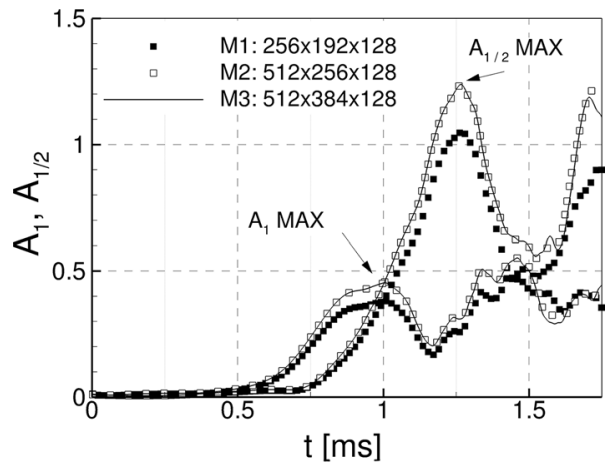

mean values of $|S|$ are almost symmetric across the mixing layer while the SDRs are not. This is because in some realizations the spark is placed almost exactly on the interface between the fuel and the oxidizer stream, where the mixture fraction gradients are very large. Such events increase the mean value to the level that cannot be compensated by other events due to small number of samples. Worth noticing is that most of the sparks is placed in the fuel rich locations, but it should be reminded that the local conditions in the spark region changes dynamically as the vortical structures continuously evolve in the flow (see Fig. 8). Hence, pockets of rich mixture are not constantly present at the ignition location. 
Analysis of the time sequences of the obtained results shows that in the region where the strain rate is high $(-2<y / \delta<2)$ the sparks "experience" significantly different mixture compositions.

\section{Results}

\subsection{Spark Ignition Scenario}

Once the spark is turned on, a spherical region of increased energy appears in the flow domain. This is shown in Fig. 8 presenting the location and size of the spark (black circle), vorticity magnitude contours and temperature iso-surfaces during the ignition process at four selected time instants. Neither the shape of the spark nor its intensity, represented by varying colour inside the black circle, is affected by the flow field in the neighbouring vortices. It can be seen that along with the increasing spark energy, the region in its vicinity becomes hotter and high temperature values appear. Unlike the spark, the temperature iso-surfaces, which initially also had spherical shape, are quickly deformed by the surrounding vortices and it seems as if they were washed away from the spark surface. The temperature rises quickly and at $t=1.25 \mathrm{~ms}$ the flame engulfs the whole spark region and spreads in its vicinity, see Fig. 8c. It can be seen that the temperature iso-surfaces are much 'smoother' than the vorticity contours. Furthermore, the vorticity inside the hot region has significantly smaller values than on the boundary of the flame. This can be attributed to the fact that for a higher temperature, the molecular viscosity increases, and thus, the local Reynolds number decreases and turns out to have a stabilising effect.

If the chemical reactions are activated before the spark is switched-off, the flame kernel becomes self-propagating by spreading depending on the local mixture composition and being torn and stretched by the neighbouring vortices. Similar situation was considered in previous study (Wawrzak and Tyliszczak 2019) as successful ignition scenario No. (1). In the present paper we will stay with this notation. When the spark is placed outside the flammable region $\left(P_{f}=0\right)$, the heat produced by the spark decays without initiating the chemical reactions. It may happen that even if the spark overlaps with flammable mixture regions, the increase of the temperature is insufficient to initiate the reactions. Moreover, one should note that the failed ignition can be also observed when the spark is placed close or inside the region overlapping the stoichiometric mixture. Such a location of the spark can be only seemingly favourable for ignition as the presence of strong shear stresses or vortical structure may either destroy the flame kernel or move it to inflammable very rich/ lean flow regions. According to Wawrzak and Tyliszczak (2019), such a failed ignition scenario is termed as No. (2). However, the opposite situation is also possible, i.e. the hot fluid created in such regions can be brought by the vortices to the region with favourable conditions where the flame can develop. This is the second successful ignition scenario (No. (3)) that leads to a stable flame (Wawrzak and Tyliszczak 2019).

\subsection{Ignition Kernel Evolution}

To further investigate two different successful ignition scenarios, the flame kernel behaviour after the spark initiation had to be analysed with scrutiny. Its spatio-temporal evolution is illustrated in Figs. 9 and 10 using the high temperature isosurface coloured by the heat release for the cases with spark ignition scenarios No. (1) and No. (3), respectively. Based 
Fig. 8 Flame kernel evolution when the spark is turned on at $y / \delta=1$. The black circle shows the spark location and the colour inside the circle corresponds to the spark intensity $\dot{Q}$. The contours show the vorticity magnitude in the ' $x-y$ ' and ' $y-z$ ' cross-section planes crossing the spark. The iso-surfaces show the temperature distribution in the vicinity of the spark. The values of temperature iso-surfaces are given in the subfigure captions

on all ignition attempts we found that the ignition scenario No. (1) prevailed on the rich side of the mixing layer $(100 \%$ for $y / \delta=1, y / \delta=2 ; 87.5 \%$ at $y / \delta=0)$ and the scenario No. (3) occurred significantly more often on the lean side (83.3\% at $y / \delta=-2)$. The results presented in Figs. 9 and 10 correspond to the initial conditions labelled as S1 and S8, as discussed in Sect. 3.2. The temperature value of $1200 \mathrm{~K}$ is 'a priori' chosen to represent the shape and volume of the flames. The isolines of the vorticity magnitude at central ' $x-y$ ' and ' $z-y$ ' cross-sections are also shown to visualise the turbulent structures in the neighbourhood of the spark location (black circle). It can be seen that at the time instant $t=t_{0}$, at which the sparks reach the maximum energy, the turbulent structures already exist in the flow. Although it seems that in the cases S1 and S8, the conditions close to the spark are quite similar (see Table 2), the flame evolutions at the later stages are substantially different. In the case S1, the flame develops near its initial position and virtually does not move. In the case S8, the situation is different. In the first phase of the ignition, called later the sub-stage I, the flame kernel approaches the favourable local mixture conditions where its further expansion occurs. This process is accompanied by a rolling up vortical structure and its movement towards the left boundary. From now on, the flame development and propagation is dominant (sub-stage II). Well mixed fuel-air pockets ignite along the vortex chain which give the effect of flame stretching. This causes that the flame deforms and its shape becomes highly irregular and mostly stretched in the streamwise direction and spanwise direction. The colours representing the heat release shows in which direction the flame propagates. The temperature distribution shows that when the spark duration ends, the flame S1 extends over $15 \delta$ in $x$-direction whereas the S8 spreads over the distance smaller than $10 \delta$. In contrast, the flame S8 is wider in the $z$-direction compared to the case S1. It seems obvious that the size of the flame in case S8 is smaller, as it was ignited later, however, it will be shown momentarily that the speeds of the flame volume growth are also different in the cases S1 and S8.

To give more light on each ignition scenario Fig. 11 shows the transient evolution of the maximum heat release $\left(Q_{\mathrm{MAX}}\right)$ found at $T=1200 \mathrm{~K}$ iso-surface and the mixture fraction corresponding to these specific locations for ignition events S1 and S8. It is seen that the ignition occurs earlier for the case $\mathrm{S} 1$, as the sudden increase in $Q_{\mathrm{MAX}}$ is observed around $t=1.27 \mathrm{~ms}$ whereas for the case S8 the $Q_{\mathrm{MAX}}$ occurs at $t=1.31 \mathrm{~ms}$. At the beginning, when the kernel expansion overwhelms the convective motion, the fluctuations of $\xi$ are large compared to the later phase of the flame development. Nevertheless, the locations of the $Q_{\mathrm{MAX}}$ suggest that the flames propagate along the lean and nearly stoichiometric mixture for both ignition scenarios.

Two sub-stages identified for the ignition scenario No. (3) are further analysed in Fig. 12 showing the time evolution of the maximum temperature $\left(T_{\mathrm{MAX}}\right)$ inside the computational domain, the normalized strain rate $\left(S=|S| /|S|_{\text {MAX }}^{\mathrm{INI}}\right)$ at the locations of the mesh points where $T_{\mathrm{MAX}}$ is found, and the rate of change of a flame volume $\left(d V_{\text {FLAME }} / d t\right)$. The flame volume is defined as the sum of the volumes of computational cells in which the temperature is larger than $1200 \mathrm{~K}\left(V_{\mathrm{FLAME}}=\sum V_{\text {cell }}^{T \geq 1200 \mathrm{~K}}\right)$. Figure 12 also shows the solutions obtained applying the mesh M3 (case S8-M3). As can be seen, the profiles almost overlap, which to some extent justifies the use of 'no model' approach as the sub-filter activity in 


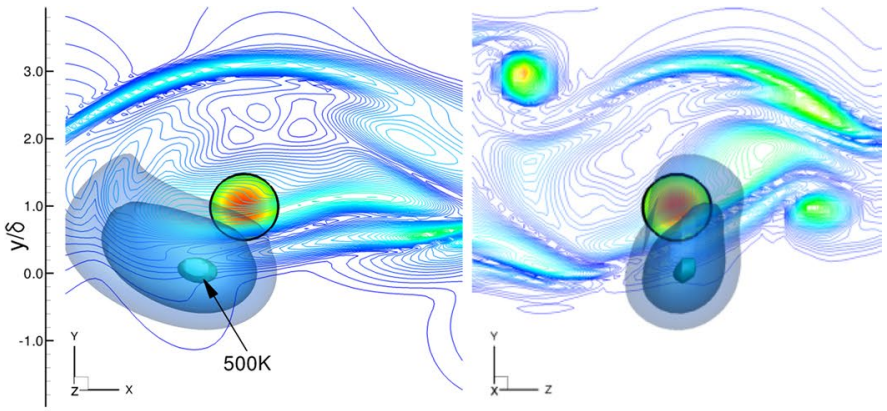

(a) $t=1.15 \mathrm{~ms}, T_{\text {iso }}=350,400,500 \mathrm{~K}$

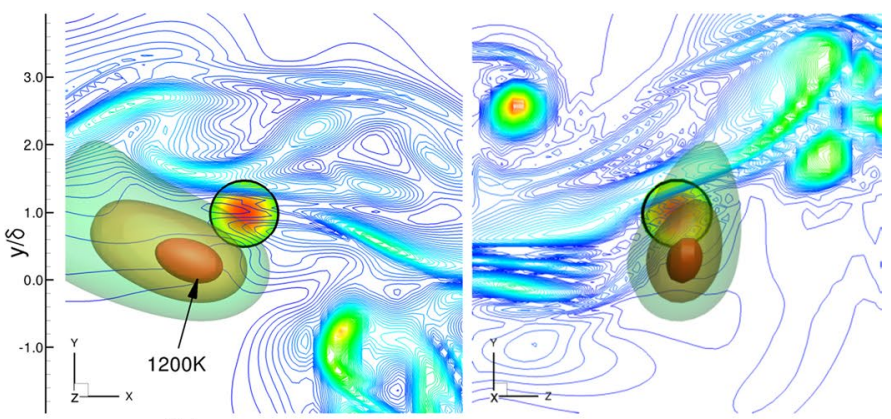

(b) $t=1.25 \mathrm{~ms}=t_{0}, T_{\text {iso }}=600,900,1200 \mathrm{~K}$

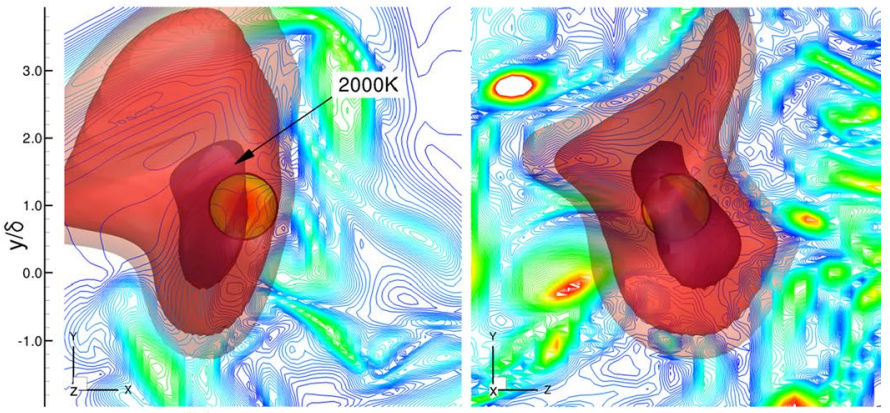

(c) $t=1.35 \mathrm{~ms}, T_{\text {iso }}=1000,1500,2000 \mathrm{~K}$

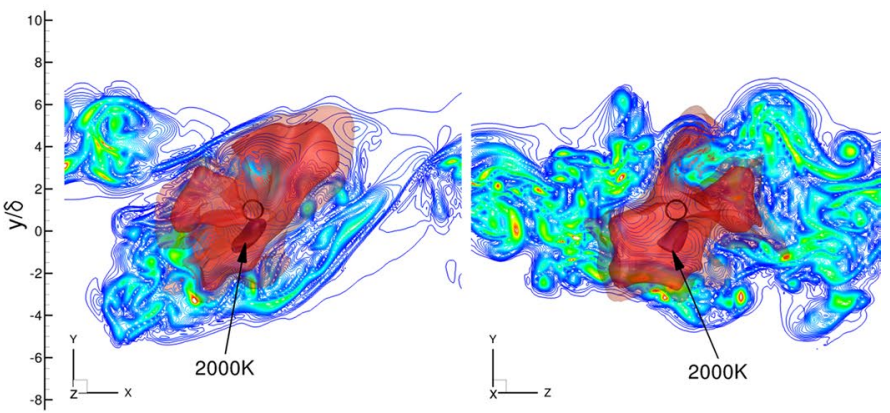

(d) $t=1.50 \mathrm{~ms}=t_{0}+\Delta_{t} / 2($ enlarged view $)$ 


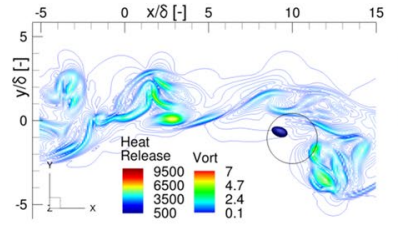

(a) $t=1.25 \mathrm{~ms}=t_{0}$

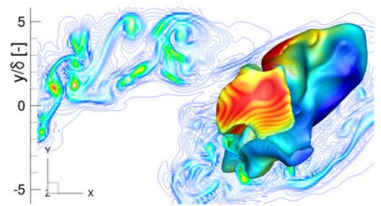

(c) $t=1.50 \mathrm{~ms}=t_{0}+\Delta_{t} / 2$
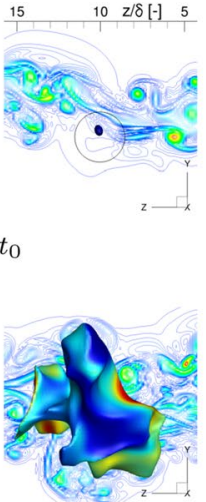

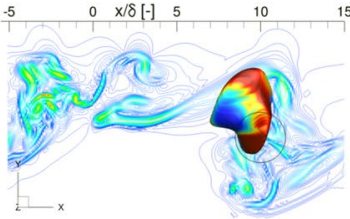

(b) $t=1.35 \mathrm{~ms}$
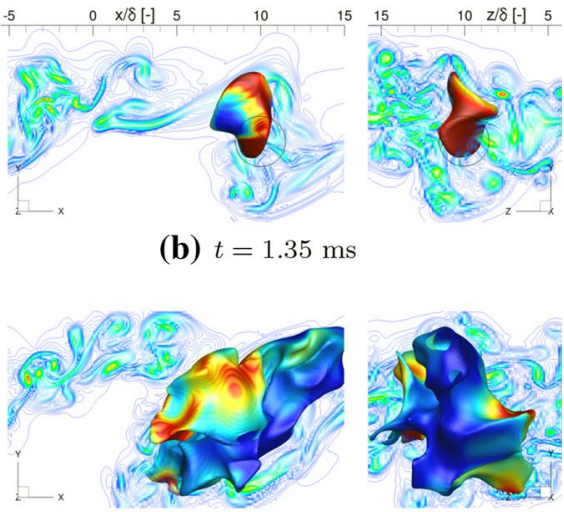

(d) $t=1.55 \mathrm{~ms}$

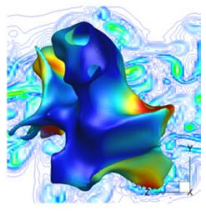

Fig. 9 Instantaneous temperature iso-surfaces $(1200 \mathrm{~K})$ coloured by heat release and vorticity magnitude isolines in the central cross sections at subsequent time instants following the successful ignition scenario with the spark located at $y / \delta=-1$. Results for the case S1

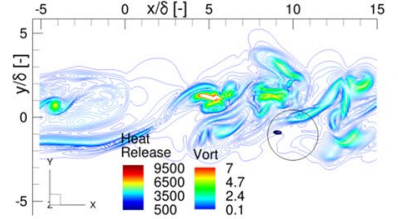

(a) $t=1.25 \mathrm{~ms}=t_{0}$

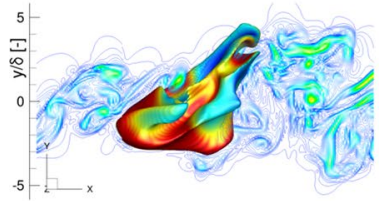

(c) $t=1.50 \mathrm{~ms}=t_{0}+\Delta_{t} / 2$
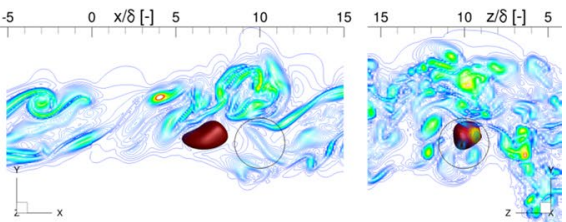

(b) $t=1.35 \mathrm{~ms}$
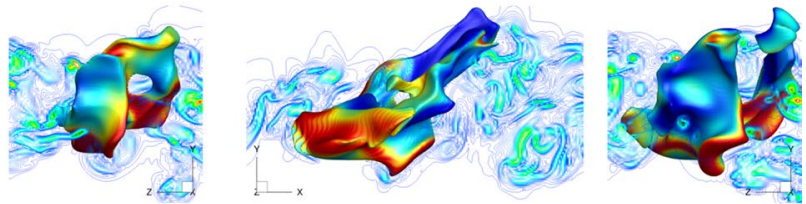

(d) $t=1.55 \mathrm{~ms}$

Fig. 10 Instantaneous temperature iso-surfaces $(1200 \mathrm{~K})$ coloured by heat release and vorticity magnitude isolines in the central cross sections at subsequent time instants following the successful ignition scenario with the spark located at $y / \delta=-1$. Results for the case S8

the vicinity of very intense reactions is at very low level. The $T_{\text {MAX }}$ profile shown for the case S1 (scenario No. (1)) characterizes a steep increase during the sub-stage I, followed by a small temperature drop. In the sub-stage II the temperature stabilizes and the flame volumes grows rapidly. A significantly different behaviour is reflected through the two-steps rise of $T_{\mathrm{MAX}}$ observed for the case S8 (scenario No. (3)). Here, the temperature initially increases to a moderate level (sub-stage I), it remains there for a short time or slightly decreases, and then suddenly rises (sub-stage II) and stabilizes similarly as in the case S1. Such a two-step flame evolution is attributed to unfavorable flow conditions in the place/ time of the spark generation (sub-stage I) and the movement of hot pockets of gas towards a region where the chemical reactions can proceed (sub-stage II). Comparison of the $S$ evolution (Fig. 12b) shows that delay of the ignition in scenario No. (3) in the case S8, is caused by large velocity gradients in the region of the flame kernel formation. Although at the 
Fig. 11 Maximum heat release and the mixture fraction at $T=1200 \mathrm{~K}$ isosurface in a function of time. The results for the cases $\mathrm{S} 1$ and $\mathrm{S} 8$ with the spark located at $y / \delta=-1$

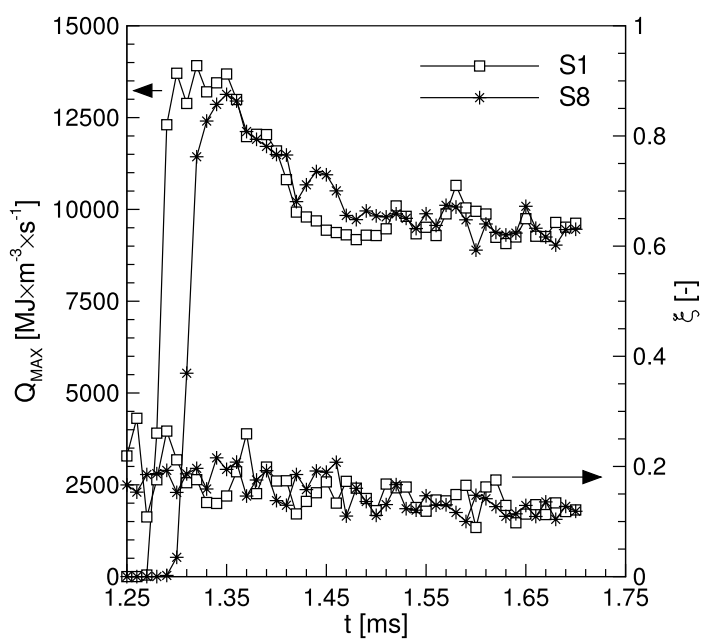

initial moment of the spark generation the strain rate for the case S8 is approximately two times smaller than for S1 (see Table 2), later, in the sub-stage I it significantly grows and its maximum is 1.3 times larger than the maximum of $S$ observed for the case S1. Large velocity gradients that contribute to $S$ are partially caused by density differences due to the spark generation and partially by the movement of strong vortical structure close to it (see Fig. 10). Regarding the difference in the flame volumes in the cases $\mathrm{S} 1$ and $\mathrm{S} 8$, the $V_{\text {FLAME }}$ is approximately two times smaller for S8 during the entire simulation time. Figure $12 \mathrm{c}$ shows the speed of the flame volume growth $d V_{\mathrm{FLAME}} / d t$ during the ignition and at the later phase. The red lines represent the linear fit of the results and they indicate that before the $T_{\mathrm{MAX}}$ stabilizes the flame in the case $\mathrm{S} 1$ expands slightly faster, and on the other hand, when the $T_{\mathrm{MAX}}$ becomes equal for both the cases then the flame growth is more intense for the case S8, though the difference is not very large. Worth noticing is that when the $T_{\mathrm{MAX}}$ is stable the $d V_{\text {FLAME }} / d t$ is approximately two times bigger than during the transient phase.

In the following the results obtained from the ignition trials for different initial solutions (S1, S4, S13 and S14) at the spark locations $y / \delta=0$ and $y / \delta=-2$ are compared. Figure 13 shows the ratios $T_{\mathrm{MAX}}^{(1)} / T_{\mathrm{MAX}}^{(3)}$ and $V_{\mathrm{FLAME}}^{(1)} / V_{\mathrm{FLAME}}^{(3)}$, where the superscript denotes the ignition scenario. The results at three time instances are presented, $t=1.35 \mathrm{~ms}$, $t=1.50 \mathrm{~ms}$ and $t=1.65 \mathrm{~ms}$. They are denoted by right triangle, left filled triangle and diamond, respectively. The first time moment corresponds to the time shortly after the spark reached the maximum energy, the second one matches the end of the spark duration, and the last one is the time when the maximum temperatures stabilize. Initially, at $t=1.35 \mathrm{~ms}$ the $T_{\mathrm{MAX}}$ and $V_{\mathrm{FLAME}}$ ratios are large, which means that the flame volumes differ substantially. At the later time, as the temperature maxima stabilize and the ratios $T_{\mathrm{MAX}}^{(1)} / T_{\mathrm{MAX}}^{(3)}$ approach to one, the ratios $V_{\text {FLAME }}^{(1)} / V_{\text {FLAME }}^{(3)}$ decrease. Nevertheless, in all analysed cases, the flame volumes in the ignition events following the scenario No. (1) were larger and the ratios $V_{\text {FLAME }}^{(1)} / V_{\text {FLAME }}^{(3)}$ were in the range 2-4.

\subsection{Flame in Mixture Fraction Space}

Structure of the flame in mixture fraction space is presented in Fig. 14. The scatter plots show the temperature coloured by the mass fraction of $\mathrm{OH}$ radical for three cases, which 
Fig. 12 Maximum temperature $\left(T_{\mathrm{MAX}}\right)$ in the computational domain (a), the normalized strain rate $(S)$ at the $T_{\text {MAX }}$ locations (b), and the gradient of the flame volume (c) as a function of time. The red lines in (c) are the linear approximations. The results are for the cases S1, S8 and S8-M3 with the spark localised at $y / \delta=-1$

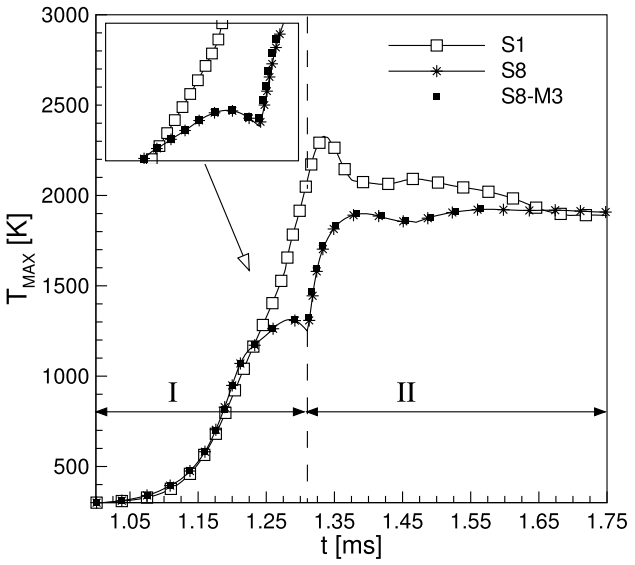

(a)

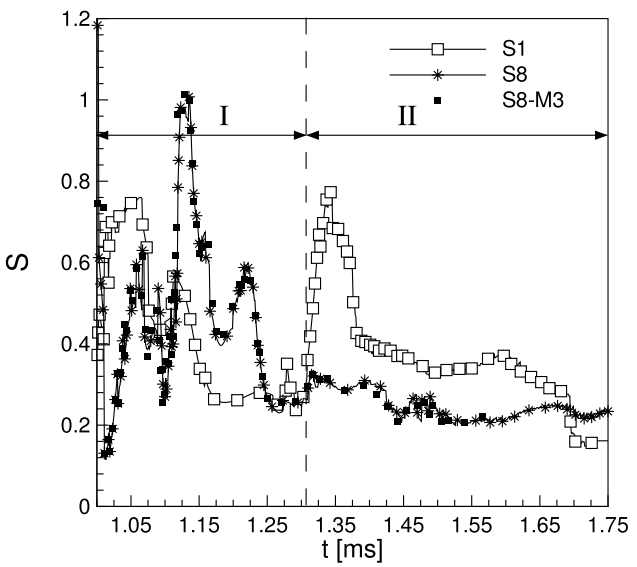

(b)

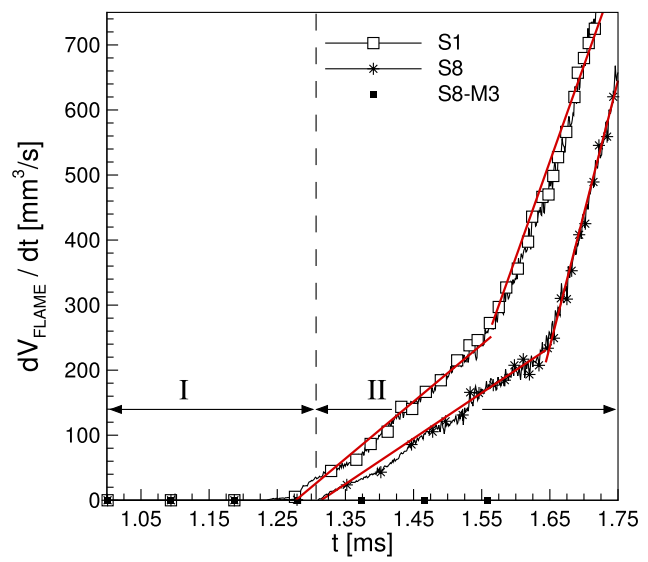

(c) 
Fig. 13 Maximum temperature and flame volumes ratios for different ignition events

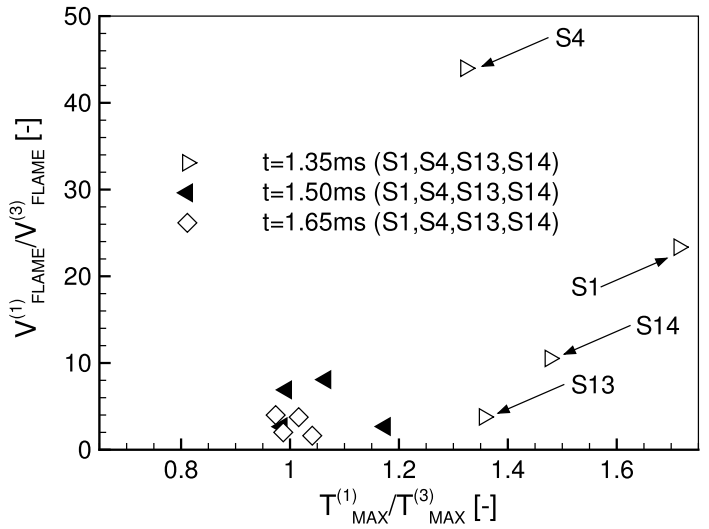

differ by the spark location $(y / \delta=0, y / \delta=-1)$ and randomness of the initial flow field (S0, S1). It can be seen that both these factors have a strong impact on the solution. The ignition was successful for both the cases but the initial flame development scenarios were significantly different. This can be concluded from the fact that the points with high temperature values are located in different parts of $\xi$-space. It can be seen that at $t=t_{0}$ the flame begins to develop only for the case S1 with the spark at $y / \delta=0$. The increased $\mathrm{OH}$ mass fraction values indicate the proceeding chemical reactions. The most interesting seems to be the case S0, with the spark located at $y / \delta=0$, for which at $t=t_{0}$ the majority of the 'hot points' lies in a very rich region, even close to $\xi=1$. As can be seen in Fig. $14 \mathrm{~b}$ when the time passes they all move towards the lean fuel side where they fit under an envelope typical for the flamelet type solution. A sample flamelet profile for the case with $Y_{\mathrm{H}_{2}}=0.1, Y_{\mathrm{N}_{2}}=0.9, T=300 \mathrm{~K}$ for $\xi=1.0$ and $Y_{\mathrm{O}_{2}}=0.1, Y_{\mathrm{N}_{2}}=0.9, T=1200 \mathrm{~K}$ for $\xi=0.0$ is represented in Fig. $14 \mathrm{~b}$ by the down oriented black triangles.

Concerning the results at particular time moments, one should realize that the points with high temperature on the rich side at $t=t_{0}$ (open circles) cannot be related to the heat release and chemical reactions as the flame cannot exist above the flammability limits. The $\mathrm{OH}$ concentration is at a very low level that also indicates that the flame does not

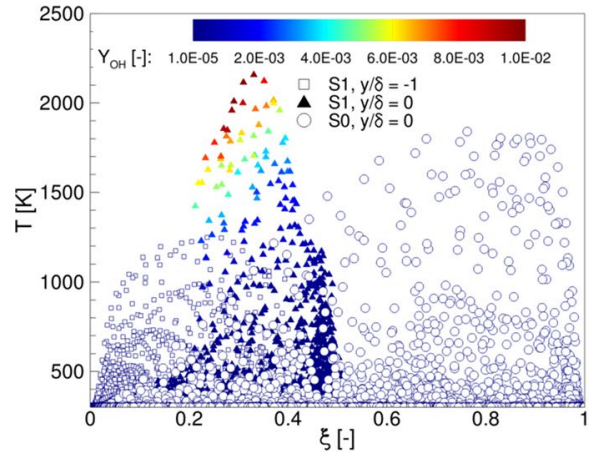

(a) $t=t_{0}$

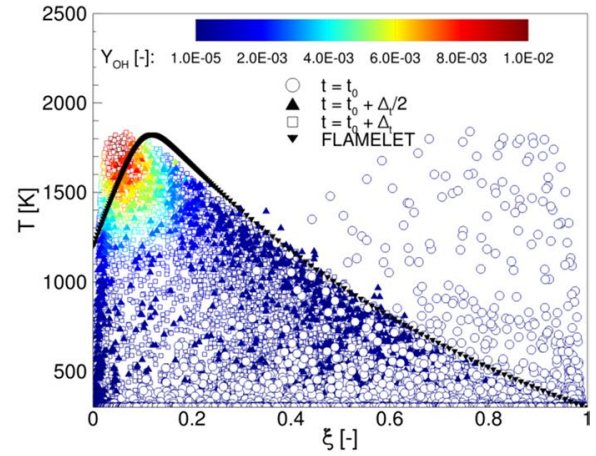

(b) case $\mathrm{S} 0, y / \delta=0$

Fig. 14 Scatter plots of the temperature coloured by the $\mathrm{OH}$ mass fraction for the cases S0 and S1 at $t=t_{0}$ (a) and for the case S0 for three time instants (b) 
evolve in this range of $\xi$. Hence, it seems that this situation results from diffusive heat transfer and movement of hot gases from the burning region towards the upper fuel-rich side of the domain. The latter effect is dominant and is caused by strong mixing, which is manifested by large values of the scalar dissipation rate (SDR, $\chi=2 D \nabla \xi \cdot \nabla \xi$ ). Its conditional values $\left(\left.\chi\right|_{T>1200 \mathrm{~K}}\right)$ at the selected time instants are shown in Fig. 15. Additionally, the conditional mass fraction of the $\mathrm{OH}$ and heat release values are presented in Figs. 16 and 17. It can be seen that at $t=t_{0}$, none of these two quantities exist on the rich side, whereas the SDR reaches high levels mainly for $\xi>0.5$. This unequivocally means the presence of intense mixing (large mixture fraction gradients $\rightarrow$ large $\chi$ ) but no chemical reactions. At the later times, when the flame has partially developed, the maximum values of the conditional SDR concentrate only in the vicinity of $\xi_{\mathrm{ST}}=0.225$ and they decrease with time. Based on the scatter plots showing $\mathrm{OH}$ radicals and heat release, one can deduce that after the spark is switched-off, the flame expands in physical space mainly along the lean and near stoichiometric regions, which is consistent with results presented in Fig. 11 and findings of Chakraborty et al. (2007).

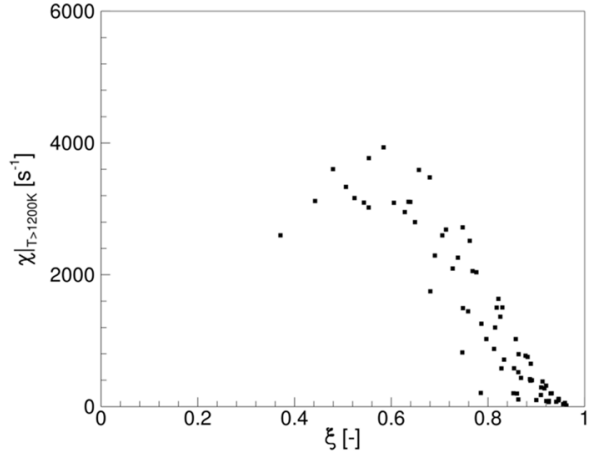

(a) $t=t_{0}=1.25 \mathrm{~ms}$

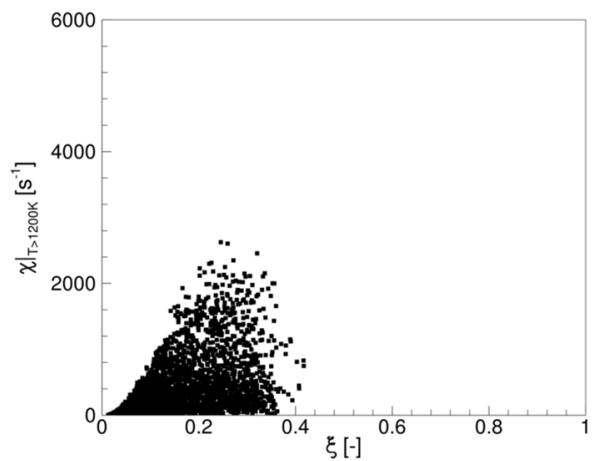

(c) $t=1.50 \mathrm{~ms}$

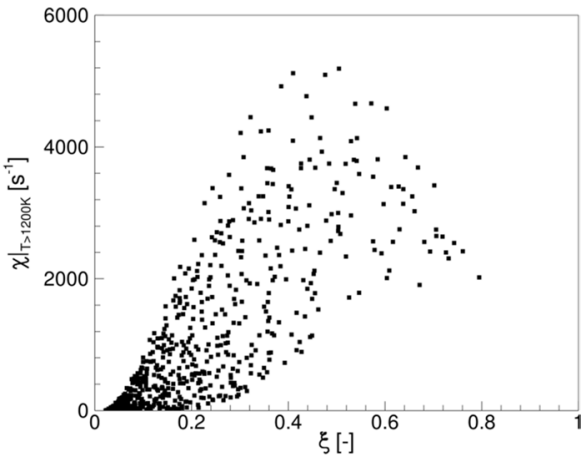

(b) $t=1.30 \mathrm{~ms}$

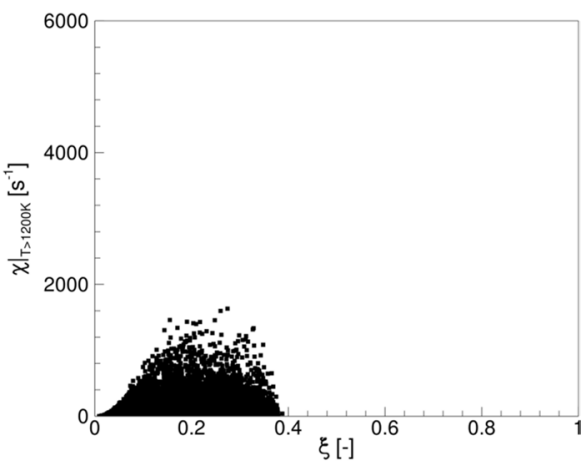

(d) $t=1.75 \mathrm{~ms}$

Fig. 15 Scatter plots of the scalar dissipation rate conditioned by $T>1200 \mathrm{~K}$ for various time instants. The results are for the case $\mathrm{S} 0$ with the spark localised at $y / \delta=0$ 


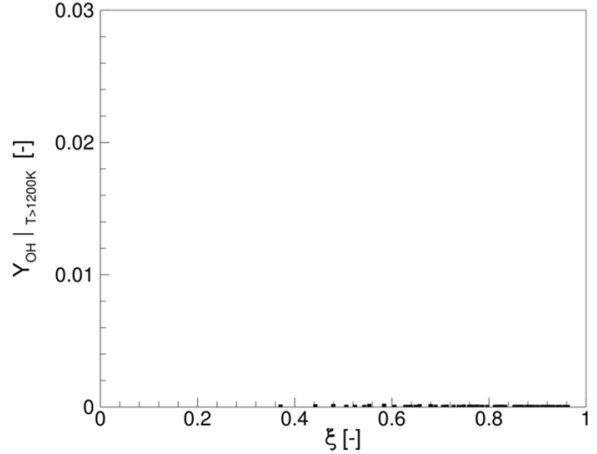

(a) $t=t_{0}=1.25 \mathrm{~ms}$

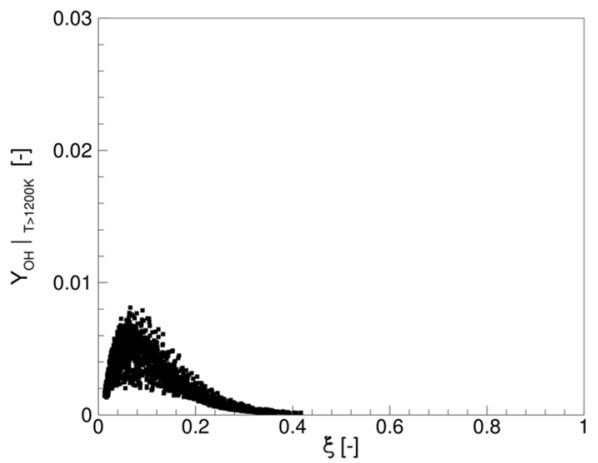

(c) $t=1.50 \mathrm{~ms}$

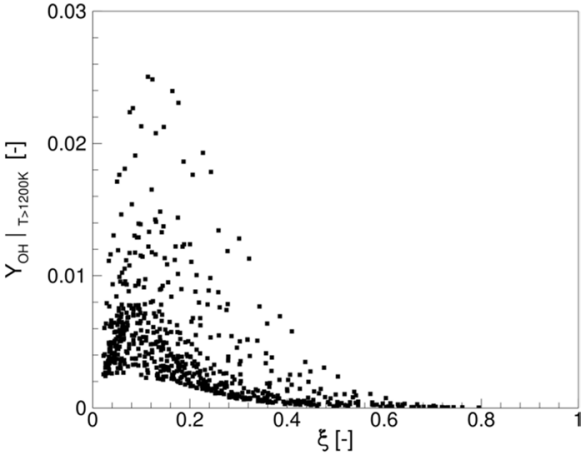

(b) $t=1.30 \mathrm{~ms}$

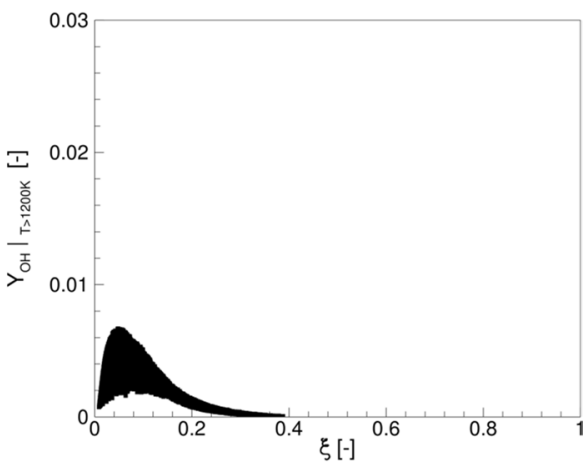

(d) $t=1.75 \mathrm{~ms}$

Fig. 16 Scatter plots of the $\mathrm{OH}$ mass fraction conditioned by $T>1200 \mathrm{~K}$ for various time instants. The results are for the case $\mathrm{S} 0$ with the spark localised at $y / \delta=0$

\subsection{Spark Ignition Probability}

Finally, based on 16 different initial fields we focus on the probability of the flame kernel generation $\left(P_{k e r}\right)$ and ignition $\left(P_{i g n}\right)$ across the mixing layer. For each of 224 ignition attempts ( 16 trials $\times 7$ locations $\times 2$ ignition times) the maximum temperature and the mass fraction of $\mathrm{OH}$ species were monitored. The flame kernel formation was classified as successful if the temperature increased high enough to initiate the chemical reactions and the $Y_{\mathrm{OH}}$ raised over $2 \times 10^{-4}$. This condition was chosen in analogy to the one used in monitoring the occurrence of auto-ignition spots. If after the spark was switched-off the high temperature persisted and the flame volume continuously grew such an event was treated as successful ignition. In contrast, if the temperature and $\mathrm{OH}$ content dropped down shortly after the spark deposition, the ignition was treated as failed. There were also ignition events in which the flame kernel has not been generated at all, i.e. the $\mathrm{OH}$ was not produced. In the cases in which the above depicted signs of ignition were observed the simulations were continued for the time period $3 \Delta_{t}$, otherwise they lasted only for $1.5 \Delta_{t}$ from the spark initiation. We compare the results analysed previously for the ignitions at the time $t_{0}=t_{A_{1 / 2} \mathrm{MAX}}$ and also the solutions for the cases with the sparks turned-on at the earlier time with $t_{0}=t_{A_{1} \mathrm{MAX}}$, i.e. before the small vortices rolled up and transformed to the large structures. 


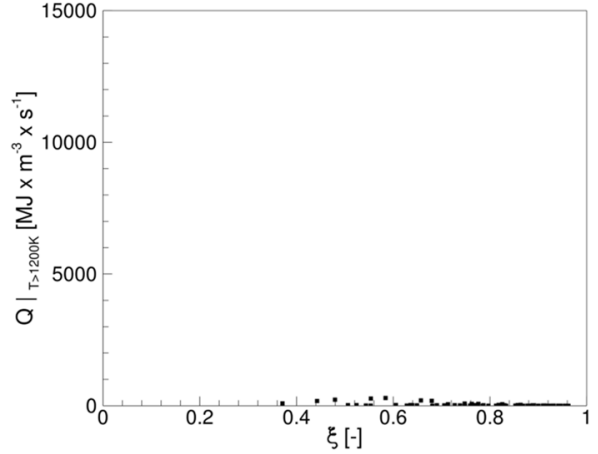

(a) $t=t_{0}=1.25 \mathrm{~ms}$

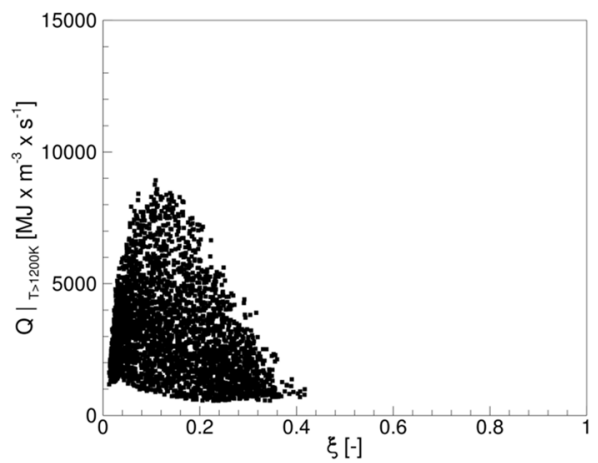

(c) $t=1.50 \mathrm{~ms}$

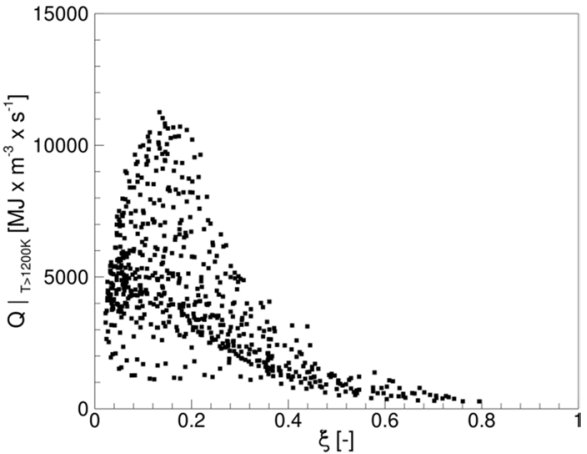

(b) $t=1.30 \mathrm{~ms}$

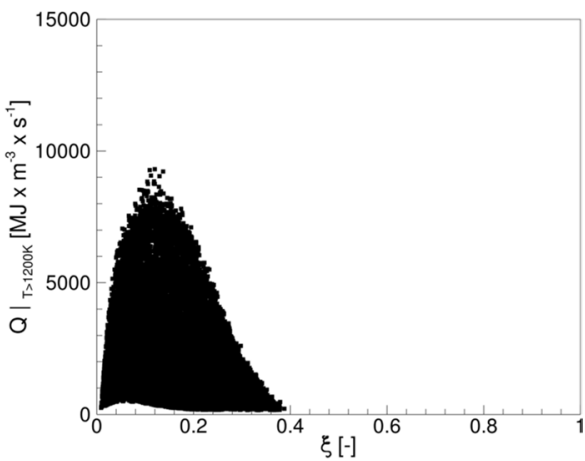

(d) $t=1.75 \mathrm{~ms}$

Fig. 17 Scatter plots of the heat release values conditioned by $T>1200 \mathrm{~K}$ for various time instants. The results are for the case $\mathrm{S} 0$ with the spark localised at $y / \delta=0$

Figure 18 shows the profiles of mean mixture fraction $\left(\xi(y)_{\text {MEAN }}\right)$ variations across the mixing layer, which were calculated by spatial averaging in the ' $x-z$ ' planes. The length of the horizontal solid line corresponds to the spark size $\Delta_{\mathrm{s}}=3 \delta$. It shows that the regions of two neighbouring sparks overlap. Although the initial fields are characterised by the same global parameters $\left(\operatorname{Re}_{\delta}, \operatorname{Re}_{\lambda}, T_{i}, \eta\right.$, mean velocities), it can be seen that the profiles of $\xi(y)_{\text {MEAN }}$ are very similar but not exactly the same and differences increase with the time. They would be identical only in the case of infinite domain in the $x$ and $z$-directions. The fact that depending on the initial velocity disturbances the evolved vortical structures had slightly different statistical properties allowed us to presume that at the ignition times the flow fields in the vicinities of the sparks differed from each other. It was verified by careful checking of every single flow field. Hence, in each case, the sparks experienced different flow conditions, even if they were placed in the same spatio-temporal locations. This led to failed or successful ignition with different flame development phases, as was discussed in Sect. 4.2. Note that the relatively small number of trials slightly diminishes credibility of the probability analysis, however, it gives an idea on how sensitive the ignition event is on the location of the spark close to the mixing layer. The monitored ignition event is binary ('no', 'yes'), so its variance is given by $c(1-c)$, where $c$ is the number of 'yes' divided by the total number of tests $N$. The statistical error is given by $\sqrt{(c(1-c))} / \sqrt{(N)}$, so, the 


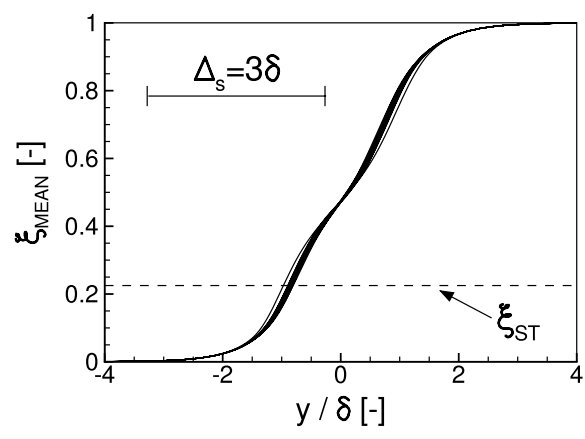

(a) $\mathrm{t}=0.75 \mathrm{~ms}$

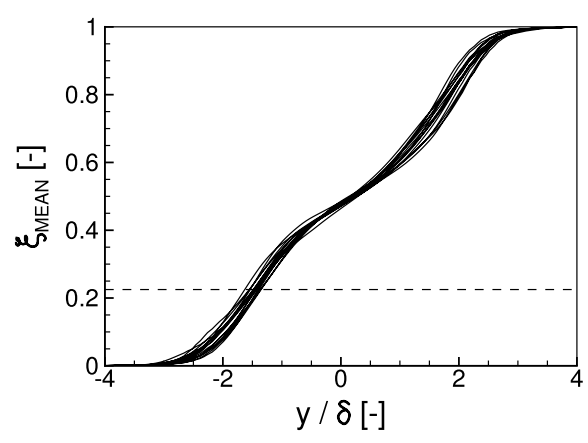

(b) $\mathrm{t}=1.00 \mathrm{~ms}$

Fig. 18 Mean mixture fraction profiles across the mixing layer for the cases S0-S15

statistical uncertainty is the highest for $P_{i g n}=0.5$ and equals to 0.125 . It is smaller at lower and high probabilities.

The computed probabilities are presented in Fig. 19 where the bars denote the statistical error at particular probability values. It can be seen that $P_{k e r}$ as well as $P_{i g n}$ distributions manifest the same trend, they are not symmetric in $y$-direction and vary from one point to another even if the spark regions overlap. Comparing directly their values it can be seen that there are only a few points in which $P_{k e r}<P_{i g n}$, which means that most of the flame kernels successfully evolved in time and space. Substantial sensitivity of the $P_{k e r}$ and $P_{i g n}$ on the spark localization is in line with Mastorakos (2009) and confirms that this process is very much dependent on local flow regimes. The highest $P_{i g n}$ occurs in the centre of the domain where the mean velocity is close to zero and it decreases drastically when the spark is moved outward. It falls to zero when sparks are placed at $y / \delta \geq \pm 4$, where the mean mixture compositions are beyond the flammability limits. This is consistent with the findings of Ahmed and Mastorakos (2006) for a jet type flame. They observed that in a region close to a nozzle the flow can be ignited only if the spark is placed in a thin region of the mixing layer in which a fuel is mixed with the oxidiser. In the present study, we found that ignition probability increases for the cases with the spark initiated later $\left(t_{0}=t_{A_{1 / 2} \mathrm{MAX}}\right)$ as in this time moment the fuel and oxidiser are better mixed and the shear stresses existing in

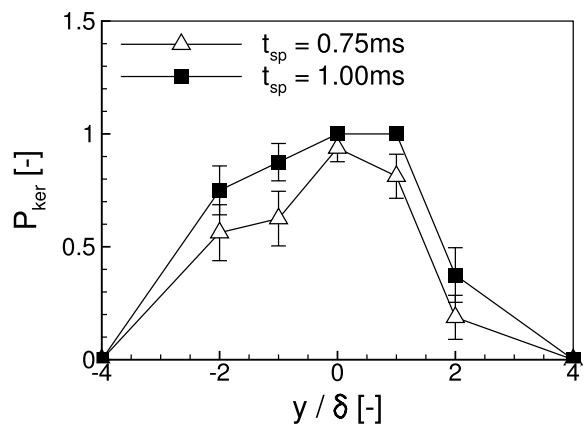

(a)

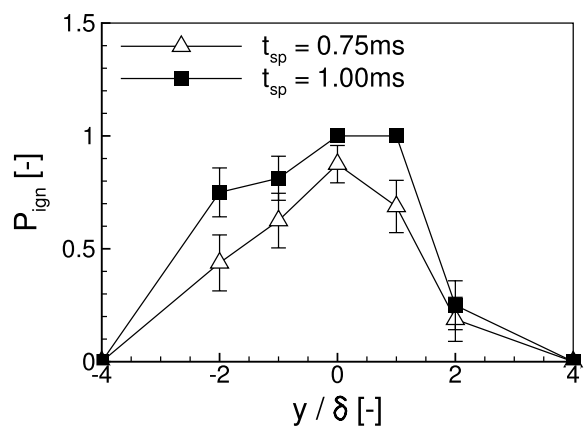

(b)

Fig. 19 Probability of flame kernel formation (a) and ignition (b) across the mixing layer 
the initial flow field become weaker. Knowing that larger vortices are present at the later time it seems that their presence does not have a significant impact on the $P_{i g n}$ distribution as it shows the same trends for $t_{0}=t_{A_{1 / 2} \mathrm{MAX}}$ and $t_{0}=t_{A_{1} \mathrm{MAX}}$. Furthermore, we observed that:

1. When the ignition occurs in the region $(-y / \delta, y / \delta)$, the kernels formed at leaner mixtures are less resistant than these at the fuel-rich side. This is somewhat surprising as the mean strain rates are similar on both sides (see Table 2) while the mean mixture composition in $(-y / \delta, 0)$ is closer to the stoichiometric regimes-see Fig. 18 and Table 2.

2. When the ignition occurs at $y / \delta \pm 2$ then the $P_{i g n}$ drastically drops down on the rich side, whereas for the sparks located on the leaner side, the $P_{i g n}$ stays at a comparable level as in the centre of the domain.

3. Regarding the ignition probability in view of the mean values presented in Table 2 it can be said that the strain rate and the scalar dissipation rate play less important role than the mixture composition. The $P_{k e r}$ and $P_{i g n}$ are the definitely higher on the fuel rich side $\left(2.5 \xi_{\mathrm{ST}}<\xi<3.3 \xi_{\mathrm{ST}}\right)$.

\section{Conclusions}

The paper presented LES studies of spark ignition process in the temporally evolving mixing layer formed between the hydrogen/nitrogen mixture and air. The performed test computations and analyses of the sub-filter scale quantities showed that the applied mesh was sufficiently dense to apply LES with 'no model' approach for the reactive terms. The aim of the study was to analyse the flame kernel behaviour and its topology during the spark initiation and soon after it is switched-off. We focused on the flames following two different ignition scenarios (No. (1) and No. (2)) that depend on the localization of the spark with respect to the vortex structures rolling-up in the mixing layer. It was shown that some sparks led to a sudden and high temperature rise resulting in a quick development of the flame whose initial shape was torn and stretched by the vortical structures. In such a case, the flame kernel was formed near the spark position. Different ignition scenario was observed mainly for the spark locations shifted from an easily ignitable region. If this was the case, the flame kernel formation was significantly delayed and two sub-stages of the ignition process were identified: (1) the temperature raised only to some intermediate level at which the chemical reactions could not proceed; (2) the heat from the volume of the hot gas diffused and in the same time this volume was torn by the vortices towards the flow parts with ignitable mixture composition where the flame could rapidly develop. In such a situation, the flame shape was very irregular and strongly dependent on the flow conditions around it. It was found that in the cases when the developing flames were moved from the place of initiation, their volumes were significantly smaller compared to the volume of the flames growing near the spark localization. It was found that the scenario No. (1) prevailed when the spark were placed if the fuel rich locations while the scenario No. (3) dominated on the lean side. In all successful ignition events the flames expanded mainly along the lean and near stoichiometric regions. The analysis of the solutions in mixture fraction space showed that this happens even if the spark was placed in the fuel rich side. In such events the nascent flame first propagated quickly towards the leaner conditions and then developed. 
Concerning the ignition probability, the present study confirmed the known fact that the ignition is hardly possible when the spark is placed far from the mixing layer region. The unsuccessful ignition trials were observed also for the sparks placed in the regions of ignitable mixture but in unfavourable flow conditions where surrounding vortices either destroyed the nascent flame or moved it towards non-flammable flow parts. It was found that when the spark was placed in the mixing layer and close to the initial boundary between the fuel and oxidiser, the ignition probability was asymmetric and larger on fuel richer side. The presence of larger vortices seems to have a large impact on the flame evolution but not on the ignition probability. Unlike the local composition the impact of the strain rate of the velocity field was also very limited in view of the ignition success. This aspect certainly needs more research with varying spark parameters (size, duration, energy) and substantially larger number of samples, in particular, as only then the statistical analysis can be fully credible. Nevertheless, taking into account that the sparks used in the present study were three times larger than the initial mixing layer thickness and with the energy significantly exceeding the minimum ignition energy, the presented distribution of ignition probability showed how sensitive the ignition process is to the location of the spark and local instantaneous flow regimes.

Acknowledgements This work was supported by National Science Center in Poland (Grant 2015/17/B/ ST8/03217) and statutory funds BS/PB-1-100-3010/2020/P. PL-Grid infrastructure was used to carry out the computations.

\section{Compliance with Ethical Standards}

Conflict of interest The authors declare that they have no conflict of interest.

Open Access This article is licensed under a Creative Commons Attribution 4.0 International License, which permits use, sharing, adaptation, distribution and reproduction in any medium or format, as long as you give appropriate credit to the original author(s) and the source, provide a link to the Creative Commons licence, and indicate if changes were made. The images or other third party material in this article are included in the article's Creative Commons licence, unless indicated otherwise in a credit line to the material. If material is not included in the article's Creative Commons licence and your intended use is not permitted by statutory regulation or exceeds the permitted use, you will need to obtain permission directly from the copyright holder. To view a copy of this licence, visit http://creativecommons.org/licenses/by/4.0/.

\section{References}

Ahmed, S., Mastorakos, E.: Spark ignition of lifted turbulent jet flames. Combust. Flame 146(1), 215-231 (2006)

Ahmed, S.F., Mastorakos, E.: Spark ignition of a turbulent shear-less fuel-air mixing layer. Fuel 164, 297304 (2016)

Ahmed, S., Balachandran, R., Mastorakos, E.: Measurements of ignition probability in turbulent non-premixed counterflow flames. Proc. Combust. Inst. 31(1), 1507-1513 (2007)

Birch, A., Brown, D., Dodson, M.: Ignition probabilities in turbulent mixing flows. In: Symposium (International) on Combustion, vol. 18, pp. 1775-1780. Elsevier (1981)

Branley, N., Jones, W.: Large eddy simulation of a turbulent non-premixed flame. Combust. Flame 127(12), 1914-1934 (2001)

Brown, P.N., Hindmarsh, A.C.: Reduced storage matrix methods in stiff ODE systems. J. Appl. Math. Comput. 31, 40-91 (1989)

Chakraborty, N., Cant, R.S.: Effects of lewis number on flame surface density transport in turbulent premixed combustion. Combust. Flame 158(9), 1768-1787 (2011) 
Chakraborty, N., Mastorakos, E.: Direct numerical simulations of localised forced ignition in turbulent mixing layers: the effects of mixture fraction and its gradient. Flow Turbul. Combust. 80(2), 155-186 (2008)

Chakraborty, N., Mastorakos, E., Cant, R.: Effects of turbulence on spark ignition in inhomogeneous mixtures: a direct numerical simulation (DNS) study. Combust. Sci. Technol. 179(1-2), 293-317 (2007)

Chen, Z., Ruan, S., Swaminathan, N.: Large eddy simulation of flame edge evolution in a spark-ignited methane-air jet. Proc. Combust. Inst. 36, 1645-1652 (2017)

Colin, O., Ducros, F., Veynante, D., Poinsot, T.: A thickened flame model for large eddy simulations of turbulent premixed combustion. Phys. Fluids 12, 1843 (2000)

Doom, J., Mahesh, K.: DNS of auto-ignition in turbulent diffusion $\mathrm{H}_{2}$ /air flames. In: 47th AIAA Aerospace Sciences Meeting including the New Horizons Forum and Aerospace Exposition, p. 240 (2009)

Duwig, C., Nogenmyr, K.J., Chan, C., Dunn, M.J.: Large eddy simulations of a piloted lean premix jet flame using finite-rate chemistry. Combust. Theory Model. 15(4), 537-568 (2011)

EidiAttarZade, M., Tabejamaat, S., Mani, M., Farshchi, M.: Numerical study of ignition process in turbulent shear-less methane-air mixing layer. Flow Turbul. Combust. 99(2), 411-436 (2017)

Geurts, B.: Elements of Direct and Large-Eddy Simulation. R.T. Edwards, Morningside (2004)

Hawkes, E.R., Sankaran, R., Sutherland, J.C., Chen, J.H.: Scalar mixing in direct numerical simulations of temporally evolving plane jet flames with skeletal $\mathrm{CO} / \mathrm{H}_{2}$ kinetics. Proc. Combust. Inst. 31(1), 1633-1640 (2007)

Jones, W.P., Prasad, V.N.: LES-pdf simulation of a spark ignited turbulent methane jet. Proc. Combust. Inst. 33, 1355-1363 (2011)

Jones, W., Tyliszczak, A.: Large eddy simulation of spark ignition in a gas turbine combustor. Flow Turbul. Combust. 85(3-4), 711-734 (2010)

Kolla, H., Zhao, X.-Y., Chen, J.H., Swaminathan, N.: Velocity and reactive scalar dissipation spectra in turbulent premixed flames. Combust. Sci. Technol. 188(9), 1424-1439 (2016)

Lacaze, G., Richardson, E., Poinsot, T.: Large eddy simulation of spark ignition in a turbulent methane jet. Combust. Flame 156(10), 1993-2009 (2009)

Laizet, S., Lamballais, E.: High-order compact schemes for incompressible flows: a simple and efficient method with quasi-spectral accuracy. J. Comput. Phys. 228, 5989-6015 (2009)

Lesieur, M., Staquet, Ch., Le Roy, P., Comte, P.: The mixing layer and its coherence examined from the point of view of two-dimensional turbulence. J. Fluid Mech. 192, 511-534 (1988)

Li, H.-M., Li, G.-X., Jiang, Y.-H.: Laminar burning velocities and flame instabilities of diluted $\mathrm{H}_{2} / \mathrm{CO} /$ air mixtures under different hydrogen fractions. Int. J. Hydrog. Energy 43, 16344-16354 (2018)

Martinez, D.O., Chen, S., Doolen, G.D., Kraichnan, R.H., Wang, L.-P., Zhou, Y.: Energy spectrum in the dissipation range of fluid turbulence. J. Plasma Phys. 57(1), 195-201 (1997)

Mastorakos, E.: Ignition of turbulent non-premixed flames. Prog. Energy Combust. Sci. 35(1), 57-97 (2009)

Mastorakos, E.: Forced ignition of turbulent spray flames. Proc. Combust. Inst. 36(2), 2367-2383 (2017)

McCraw, J., Moore, N., Lyons, K.: Observations on upstream flame propagation in the ignition of hydrocarbon jets. Flow Turbul. Combust. 79(1), 83-97 (2007)

Moser, R.D., Rogers, M.M.: The three-dimensional evolution of a plane mixing layer: pairing and transition to turbulence. J. Fluid Mech. 247, 275-320 (1993)

Mueller, M., Kim, T., Yetter, R., Dryer, F.: Flow reactor studies and kinetic modeling of the $\mathrm{H}_{2} / \mathrm{O}_{2}$ reaction. Int. J. Chem. Kinet. 31(2), 113-125 (1999)

Passot, T., Pouquet, A.: Numerical simulation of compressible homogeneous flows in the turbulent regime. J. Fluid Mech. 181, 441-466 (1987)

Pitsch, H., Duchamp De Lageneste, L.: Large-eddy simulation of premixed turbulent combustion using a level-set approach. Proc. Combust. Inst. 29(2), 2001-2008 (2002)

Poinsot, T., Veynante, D.: Theoretical and Numerical Combustion. R.T. Edwards, Morningside (2001)

Pope, S.B.: Turbulent Flows. Cambridge University Press, Cambridge (2000)

Pope, S.B.: Ten questions concerning the large-eddy simulation of turbulent flows. New J. Phys. 6(1), 35 (2004)

Prasad, V.N., Juddoo, M., Kourmatzis, A., Masri, A.R.: Investigation of lifted flame propagation under pulsing conditions using high-speed OH-LIF and LES. Flow Turbul. Combust. 93(3), 425-437 (2014)

Richardson, E., Chakraborty, N., Mastorakos, E.: Analysis of direct numerical simulations of ignition fronts in turbulent non-premixed flames in the context of conditional moment closure. Proc. Combust. Inst. 31(1), 1683-1690 (2007)

Rosiak, A., Tyliszczak, A.: LES-CMC simulations of a turbulent hydrogen jet in oxy-combustion regimes. Int. J. Hydrog. Energy 41(22), 9705-9717 (2016) 
Santhanam, N., Lele, S.K., Ferziger, J.H.: A robust high-order compact method for large eddy simulation. J. Comput. Phys. 191, 392-419 (2003)

Shu, C.W.: High-order finite difference and finite volume WENO schemes and discontinuous Galerkin methods for CFD. Int. J. Comput. Fluid Dyn. 17(2), 107-118 (2003)

Smith, M., Birch, A., Brown, D., Fairweather, M.: Studies of ignition and flame propagation in turbulent jets of natural gas, propane and a gas with a high hydrogen content. In: Symposium (International) on Combustion, vol. 21, pp. 1403-1408 (1988)

Triantafyllidis, A., Mastorakos, E.: Implementation issues of the conditional moment closure in large eddy simulations. Flow Turbul. Combust. 84, 481-512 (2009)

Triantafyllidis, A., Mastorakos, E., Eggels, R.: Large eddy simulations of forced ignition of a nonpremixed bluff-body methane flame with conditional moment closure. Combust. Flame 156(12), 2328-2345 (2009)

Tyliszczak, A.: A high-order compact difference algorithm for half-staggered grids for laminar and turbulent incompressible flows. J. Comput. Phys. 276, 438-467 (2014)

Tyliszczak, A.: LES-CMC study of an excited hydrogen flame. Combust. Flame 162(10), 3864-3883 (2015a)

Tyliszczak, A.: Multi-armed jets: a subset of the blooming jets. Phys. Fluids (1994-present) 27(4), 041703 (2015b)

Tyliszczak, A.: High-order compact difference algorithm on half-staggered meshes for low Mach number flows. Comput. Fluids 127, 131-145 (2016)

Tyliszczak, A., Cavaliere, D.E., Mastorakos, E.: LES/CMC of blow-off in a liquid fueled swirl burner. Flow Turbul. Combust. 92(1-2), 237-267 (2014)

Valiño, L.: A field Monte Carlo formulation for calculating the probability density function of a single scalar in a turbulent flow. Flow Turbul. Combust. 60, 157-172 (1998)

Vreman, A.W.: An eddy-viscosity subgrid-scale model for turbulent shear flow: algebraic theory and applications. Phys. Fluids 16, 3670-3681 (2004)

Wawrzak, A., Tyliszczak, A.: Numerical study of a turbulent hydrogen flame in oxy-combustion regimes. Arch. Mech. 62(2), 157-175 (2017)

Wawrzak, A., Tyliszczak, A.: Implicit LES study of spark parameters impact on ignition in a temporally evolving mixing layer between $\mathrm{H}_{2} / \mathrm{N}_{2}$ mixture and air. Int. J. Hydrog. Energy 43(20), 9815-9828 (2018)

Wawrzak, A., Tyliszczak, A.: A spark ignition scenario in a temporally evolving mixing layer. Combust. Flame 209, 353-356 (2019)

Zhang, H., Giusti, A., Mastorakos, E.: LES/CMC modelling of ignition and flame propagation in a nonpremixed methane jet. Proc. Combust. Inst. 37, 2125-2132 (2019) 